\title{
Handling of uncertainty in medical data using machine learning and probability theory techniques: a review of 30 years (1991-2020)
}

\author{
Roohallah Alizadehsani ${ }^{1}$ (D) - Mohamad Roshanzamir ${ }^{2}$. Sadiq Hussain ${ }^{3}$. \\ Abbas Khosravi ${ }^{1}$. Afsaneh Koohestani ${ }^{1}$ - Mohammad Hossein Zangooei ${ }^{4}$. \\ Moloud Abdar ${ }^{1}$. Adham Beykikhoshk ${ }^{5}$. Afshin Shoeibi ${ }^{6,7}$. Assef Zare ${ }^{8}$. \\ Maryam Panahiazar $^{9}$. Saeid Nahavandi ${ }^{1}$. Dipti Srinivasan ${ }^{10}$. Amir F. Atiya ${ }^{11}$. \\ U. Rajendra Acharya ${ }^{12,13,14}$
}

Accepted: 23 February 2021

(o) The Author(s), under exclusive licence to Springer Science+Business Media, LLC, part of Springer Nature 2021

\begin{abstract}
Understanding the data and reaching accurate conclusions are of paramount importance in the present era of big data. Machine learning and probability theory methods have been widely used for this purpose in various fields. One critically important yet less explored aspect is capturing and analyzing uncertainties in the data and model. Proper quantification of uncertainty helps to provide valuable information to obtain accurate diagnosis. This paper reviewed related studies conducted in the last 30 years (from 1991 to 2020) in handling uncertainties in medical data using probability theory and machine learning techniques. Medical data is more prone to uncertainty due to the presence of noise in the data. So, it is very important to have clean medical data without any noise to get accurate diagnosis. The sources of noise in the medical data need to be known to address this issue. Based on the medical data obtained by the physician, diagnosis of disease, and treatment plan are prescribed. Hence, the uncertainty is growing in healthcare and there is limited knowledge to address these problems. Our findings indicate that there are few challenges to be addressed in handling the uncertainty in medical raw data and new models. In this work, we have summarized various methods employed to overcome this problem. Nowadays, various novel deep learning techniques have been proposed to deal with such uncertainties and improve the performance in decision making.
\end{abstract}

Keywords Uncertainty - Bayesian inference $\cdot$ Fuzzy systems · Monte Carlo simulation · Classification $\cdot$ Machine learning

Roohallah Alizadehsani

ralizadehsani@deakin.edu.au

Extended author information available on the last page of the article 


\section{Introduction}

Machine learning is widely used in academia and industry to analyse big and complex datasets to uncover the hidden patterns and reach conclusive insights. It is well known that the performance of machine learning models has a close relationship not only with the selected algorithms but also depends on the nature of data (Schmidt et al., 2019). For example, having significant amount of missing values and noise in the data can affect the results. Indeed, such uncertain data are present in various fields such as energy systems (Gallagher et al., 2018; Soroudi \& Amraee, 2013), web (Nguyen et al., 2019), image (Shadman Roodposhti et al., 2019), disease and healthcare (Alizadehsani, 2019a, b, c; Alizadehsani et al., 2020; Arabasadi et al., 2017; Reamaroon et al., 2019), and the Internet of Things (Dilli et al. 2018). Hence, having such uncertainty in the medical data will makes the decision-making process difficult (Fig. 1).

Machine learning algorithms that can model uncertainty to reveal beneficial information for a better decision-making process will be of great use. Generally, uncertainty may be due to two reasons: data (noise) uncertainty and model uncertainty (also called epistemic uncertainty) (Gal, 2016). It is likely to have noise among labels due to measurement imprecision which may lead to aleatoric uncertainty. Meanwhile, model uncertainty can be divided into two main types: structure uncertainty and uncertainty in model parameters (Gal, 2016). In structural uncertainty, we find out the type of model structure to be used and to specify our proposed model for either extrapolating and/or interpolating. In the second type, i.e. uncertainty in model parameters, optimal model parameters are selected for more accurate predictions.

For the first time, Renée Fox conducted few studies which showed the uncertainties faced by physicians during their training (Fox, 1957, 1980). After that, other researchers too acknowledged the central problematic nature of this problem. Although in health care, the importance of uncertainty has been growing, we have limited knowledge about the solutions to these problems. The aim of this paper is to review the related studies in the domain of uncertainty quantification in the field of medical science. The results obtained showed that uncertainty is a common challenge among different raw data and various models. Moreover, most of the applied algorithms are Bayesian inference, fuzzy systems, Monte Carlo simulation, rough classification, Dempster-Shafer theory, and imprecise probability.

Fig. 1 Having uncertainty makes the decision-making process difficult

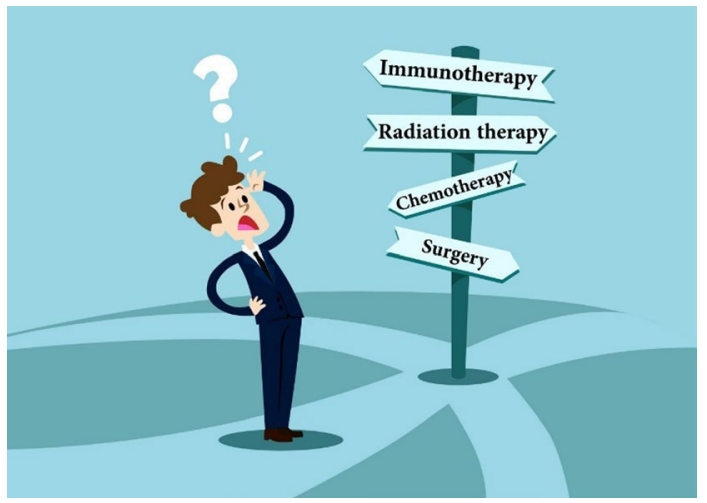


The organization of the other sections is described as follows. The search criteria used for finding papers is explained in Sect. 2. Section 3 provides more details about machine learning and probability theory methods used for handling uncertainty. Discussion and conclusion sections are presented in Sects. 4 and 5, respectively.

\section{Search criteria}

To perform this review, we performed Google scholar search for the papers published between 1 January 1991 and 31 May 2020. We have obtained most of the published papers by IEEE, Elsevier and Springer. The search keywords used for this study is Bayesian inference OR fuzzy systems OR Monte Carlo simulation OR rough classification OR Dempster-Shafer theory OR Imprecise probability AND Medical Science.

Then, about 324 papers in English language are selected and those which do not make significant impacts were removed from the list. Hence 91 high quality papers indexed in Scopus or PubMed or having a large number of citations are considered. Then, we reviewed the references of all the selected papers to find more relevant papers. Finally, 74 papers are selected in this step to be added to 91 previously selected papers. Overall, 165 papers are investigated in this review. This procedure is illustrated in Fig. 2. The scientific quality of the research overviews was assessed by the criteria listed in Table 1.

In Fig. 3, the research trend in the past 30 years is shown. It is clear from the diagram that handling uncertainty in the medical data has received more attention in recent years.

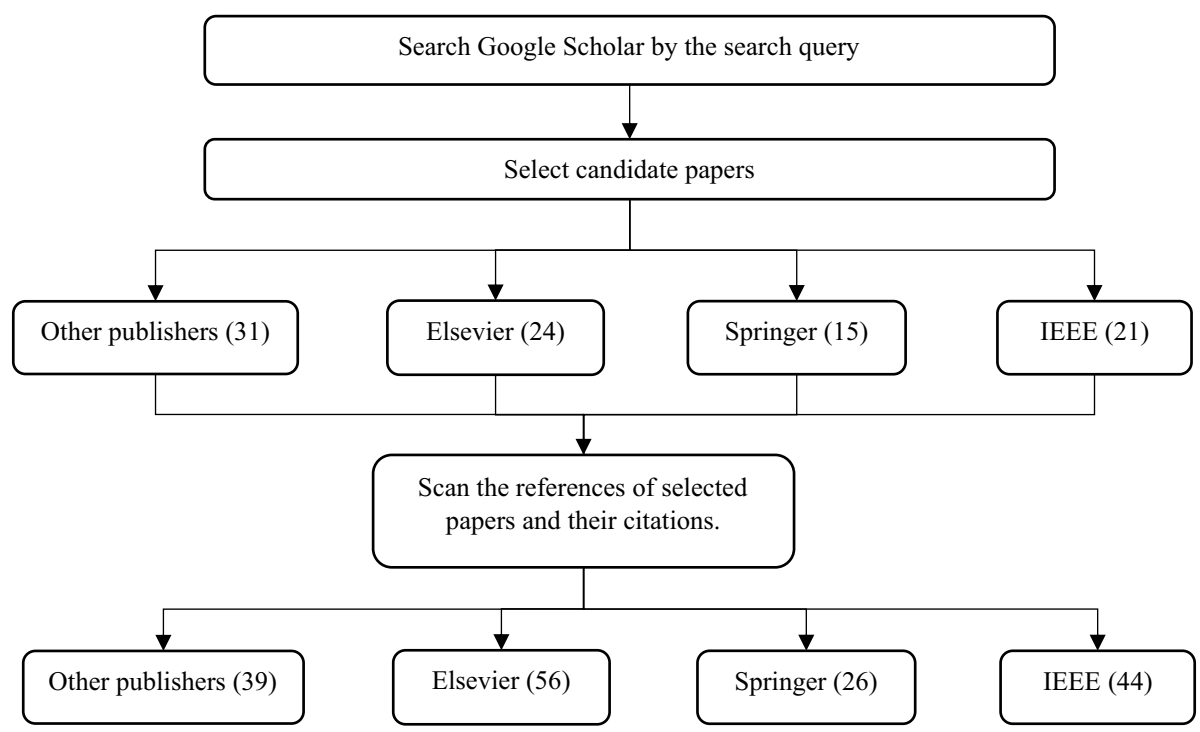

Fig. 2 Paper selection mechanism 
Table 1 The scientific quality of papers was assessed according to the following items
(1)

If the search methodology was reported
If the search methodology was comprehensive If the inclusion criteria were reported If the validity of criteria was reported If the validity was assessed properly If the findings were combined properly If the conclusions supported the reported data

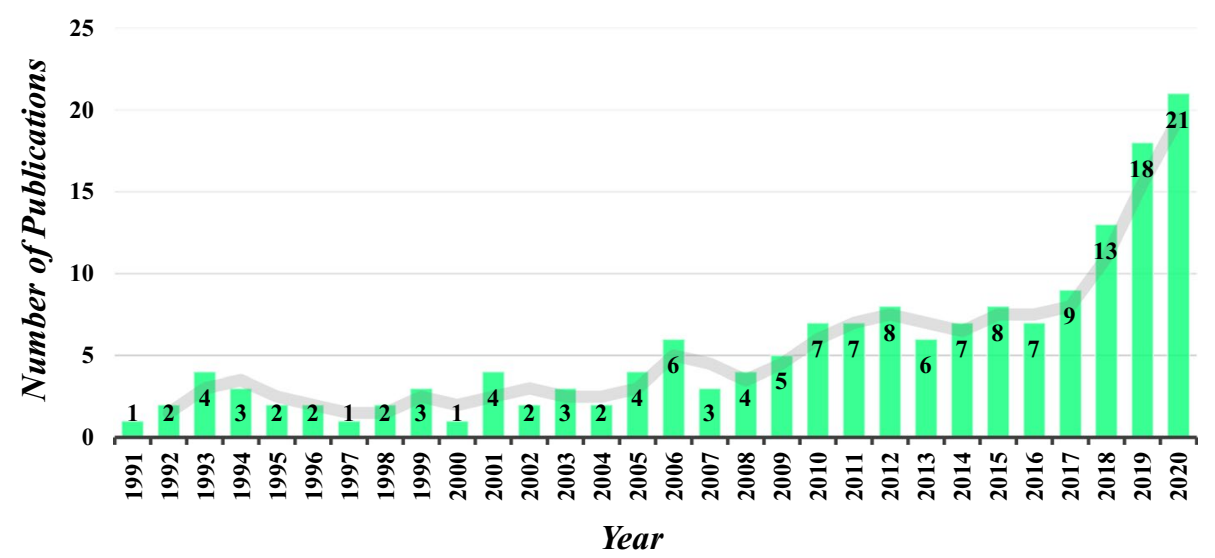

Fig. 3 Number of papers published in handling uncentainity in medical data between 1991 and 2020

Fig. 4 Most common algorithms used to handle uncertainties

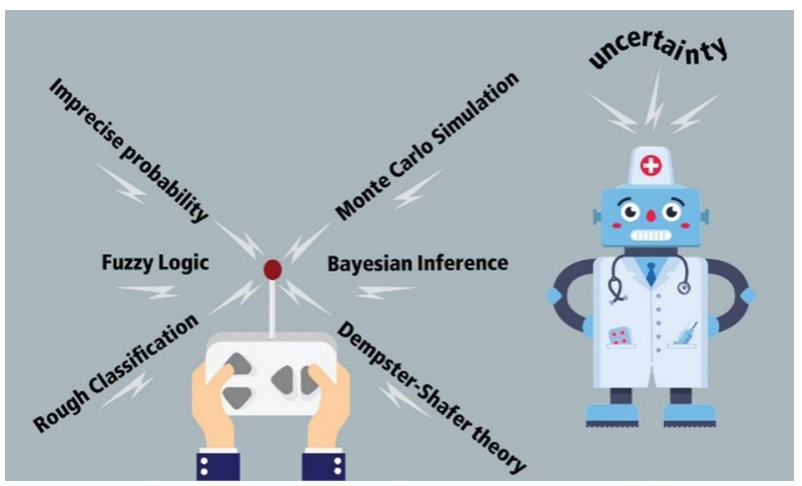

\section{Uncertainty handling algorithms in medical science}

According to the extracted papers, the most common algorithms in this field are Bayesian inference, fuzzy systems, Monte Carlo simulation, rough classification, Dempster-Shafer theory, and imprecise probability shown in Fig. 4. The most of the published papers in this field have used these algorithms to handle the uncertainties. 


\subsection{Bayesian inference (BI)}

BI is the famous statistical inference techniques which utilizes Bayes' theorem in its inference mechanism (Akkoyun et al., 2020; Corani et al., 2013; Howle et al., 2017; Kourou et al., 2020; Seixas et al., 2014; Wang et al., 2019; Watabe et al., 2014). If there is more information or evidence, the probability of a hypothesis can be updated by utilizing Bayes' theorem (Ocampo et al., 2011).

Different probabilities are described by modelling experience or knowledge base using BI which is a sort of expert system. A fundamental model is indicated by these probabilities. Based on the fundamental model, a conclusion is acquired by repeating the Bayes' theorem using the inference engine. The following equation denotes the Bayes' theorem, using the events $\mathrm{N}$ and $\mathrm{Y}$.

$$
P(N / Y)=\frac{P(Y / N) * P(N)}{P(Y / N) * P(N)+P(Y / n o N) * P(n o N)}
$$

where $\mathrm{Y}$ is particular combinations of signs and symptoms of a patient and $\mathrm{N}$ represents the fact "a patient suffers from a particular disease". By going through symptoms of Y, the probability of a person suffering from an ailment is equal to the probability of Y multiplied by the probability of the occurrence of the symptoms $\mathrm{Y}$, whether the ailment prevails or not and it is shown as:

$$
P(N / Y)=\frac{P(N \cap Y)}{P(Y)}
$$

and

$$
P(Y / N)=\frac{P(N \cap N)}{P(N)}
$$

Therefore,

$$
P(Y \cap N)=P(Y / N) * P(N)=P\left(\frac{N}{Y}\right) * P(Y)
$$

From which we get:

$$
P(N / Y)=\frac{P(Y / N) * P(N)}{P(Y)}
$$

Bayes' theorem repeatedly is applied to find a likely diagnosis of the ailment and details are described structurally as above.

A priori probability of hypothesis, given it is false or true can be utilized to calculate the probability of specific hypothesis in this specific case. Hence in the case of element of evidence, Bayes' theorem can be represented based on indicative data and ailments as follows:

$$
P(G / F)=\frac{P T * P J}{P T * P J+P M *(1-P J)}
$$

$P(G)$ depicts the likelihood of occurring an ailment and it starts with $P(G)=P J$ for each ailment. $\mathrm{P}(\mathrm{G} / \mathrm{F})$ is computed by acquiring the data from the user. The prior rule is applied when a symptomatic datum prevails. Otherwise, PT and PM are substituted by (1-PT) and 
(1-PM) by applying the same rule. A priori probability $P(G)$ is substituted by $P(G / F)$ as the outcome of each datum.

\subsubsection{Related work based on bayesian inference}

The accuracy of screening tests that are applied to identify antibodies to the human immunodeficiency virus with the help of Bayesian methods devised by Johnson et al. (Johnson \& Gastwirth, 1991). They tried to assess the incidence of the disease from the collected samples. Approximate predictive distributions for the number of future individuals that would test true positive was estimated by utilizing a novel sample or population of interest.

Robertson et al. (2010) proposed an accessible and agile adoption of Bayesian inference to design an expert system for medical diagnosis. It can be used by mid-level and low health workers in rural and remote locations. They suggested that the success of the expert system depended on the clinical interface for use in specific regions, rapid adaptation of the database, and by varying user skills.

Mazur (2012) assessed the Bayesian inference in the context of medical decision making that had three key developments: (1) need for data recognition, (2) progress of inverse probability, and (3) development of probability. The author examined Bayesian inference development from the beginning with the effusive evidence of the clinician's sign to the work of Laplace, Jakob Bernoulli, and others.

In (Ashby, 2006), Ashby examined the usage and applicability of Bayesian thinking in medical research. He reviewed the Bayesian statistics in medicine launched in 1982 to technologies associated with medical decision making such as molecular genetics, survival modeling, longitudinal modeling, spatial modeling, and evidence synthesis.

Suchard et al. (2006) proposed Bayesian posterior sampler called BAli-Phy that exploited Markov chain Monte Carlo to study phylogeny and the joint space of alignment given molecular sequence data. Their model automatically used information in shared deletions/insertions to help deduce phylogenies by utilizing more sophisticated substitution models in the alignment process.

The authors of (Mendoza-Blanco et al., 1996) utilized the theories of simulation-based techniques and missing-data analysis to develop a framework of Bayesian analysis to estimate the prevalence of immunodeficiency virus (HIV). Different practical considerations that arose from HIV screening was taken into account by their flexible techniques.

In (Huang et al., 2011), Huang et al. presented a Bayesian approach that mutually models three components. There were covariate, response, and time-to-event procedures linked thorough the random effects that demonstrated the vital individual-specific longitudinal procedures. The occurrence of CD4 covariate procedure having calculated errors in the HIV dynamic response was discussed and scrutinized disease progression by decreasing CD4/CD8 ratio to evaluate antiretroviral treatment.

The distributed and big data in the medical informatics platform framework of the Human Brain Project was analyzed by Melie-Garcia et al. (2018). They applied multiple linear regression (MLR) techniques. The Bayesian formalism offered the armamentarium necessary to execute MLR techniques for distributed big data. Their technique combined multimodal heterogeneous data coming from various hospitals and subjects around the world and recommended urbane ways that can be extendable to other statistical models.

The authors of (Johnston, 2015) devised novel generalizable theoretical and physically motivated model mitochondrial DNA (mtDNA) populations by assigning the first statistical comparison of proposed bottleneck mechanisms. 
In (Huang et al., 2010), a hierarchical Bayesian modeling technique has been presented to devise a method induced by an AIDS clinical study. Long-term virologic responses are characterized by integrating fully time-dependent drug efficacy, baseline covariates, pharmacokinetics, and drug resistance into the model. The experimental results showed that modeling virologic responses and HIV dynamics with consideration of baseline characteristics as well as time-varying clinical factors might be critical for HIV studies.

Henriquez et al. (2016) examined the Bayesian inference dilemma in medical analytics and asserted the need for probabilistic reasoning tools which is user-friendly in nature. These papers are briefly described in Table 2.

\subsubsection{Related works based on Bayesian Deep Learning}

Bayesian neural networks (BNNs) are used to add uncertainty handling in models. As it is shown in Fig. 5, BNNs learn the parameters of a random variable instead of deterministic weights. Then, backpropagation is used to learn the parameters of the random variables.

In some works, Bayesian neural network has been used to handle uncertainty (Zhang et al., 2009). We have presented few such works below.

Kendall et al. (2016) designed a visual relocalization system which has six degrees of freedom. It is a robust and real-time monocular system which is able to work indoors and outdoors scenes. It used the Bayesian convolution neural network (CNN) to obtain an accurate localization system on an outdoor dataset. Meanwhile, it has been able to detect the presence of the scene in the input images. Kendall et al. (2015) proposed a learning algorithm based on deep learning for probabilistic semantic segmentation. The main goal of the algorithm is to understand the visual scene. It also can take care of uncertainty during decision making. It has been done by Monte Carlo sampling with dropout in the test phase to generate the posterior probability of the pixel labels. By modelling uncertainty, the segmentation performance improved by about 3\%. Especially in the small dataset, the performance improved significantly because modelling uncertainty is more effective.

Although perception tasks such as object detection need human intelligence, a subsequent task that needs inference and reasoning needs more human intelligence. Nowadays, new algorithms like deep learning could extremely improve perception tasks (Alizadehsani et al., 2021; Asgharnezhad et al., 2020; Ghassemi et al., 2019, 2020; Khodatars et al., 2020; Mohammadpoor et al., 2016; Sharifrazi et al., 2020; Shoeibi et al., 2020a, b; Shoeibi, 2021; Shoushtarian et al., 2020). However, for higher-level inference, probabilistic graphical models are more powerful than other algorithms. Probabilistic graphical models are based on Bayesian reasoning. So, it seems that integrating deep learning and Bayesian models could handle both perception and inference problems which is called Bayesian deep learning. These two sections of the unified framework could boost each other. The object detection using deep learning improved the performance of a higher-level inference system. Then, the inference process feedback can enhance the object detection task. In (Wang \& Yeung, 2016), a general framework was proposed for Bayesian deep learning. Then, it was used in recommender or control systems.

In (Gal et al., 2017), an active learning framework is combined with Bayesian deep learning. Using the advantage of Bayesian convolutional neural network, they have achieved significant improvement in the existing active learning approaches, especially on high dimensional data. They tested their proposed algorithm on the MNIST dataset and also for skin cancer diagnosis. Two main branches of uncertainty modelling are aleatoric and epistemic. The former handles uncertainty in data and the later handles it in the model. 


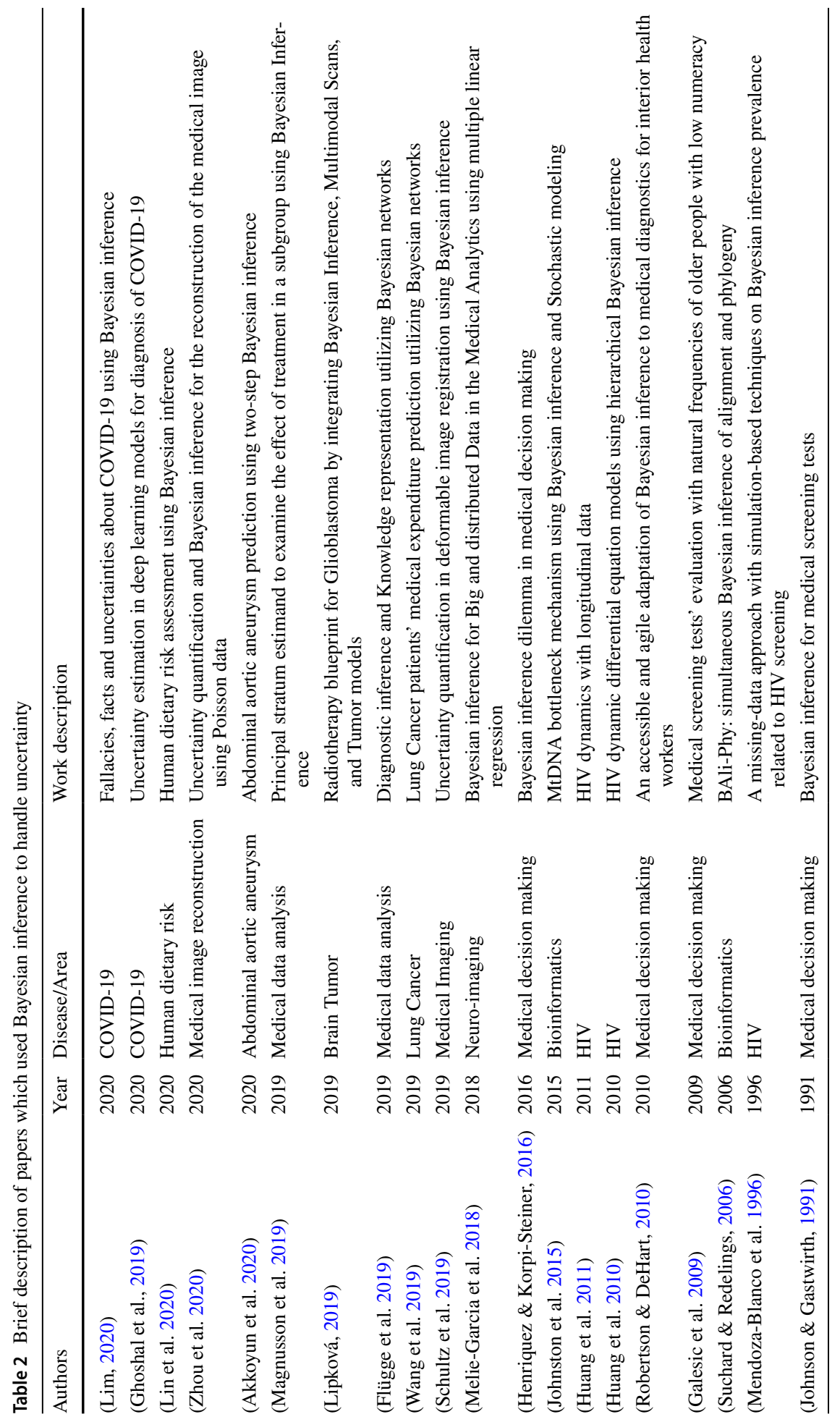



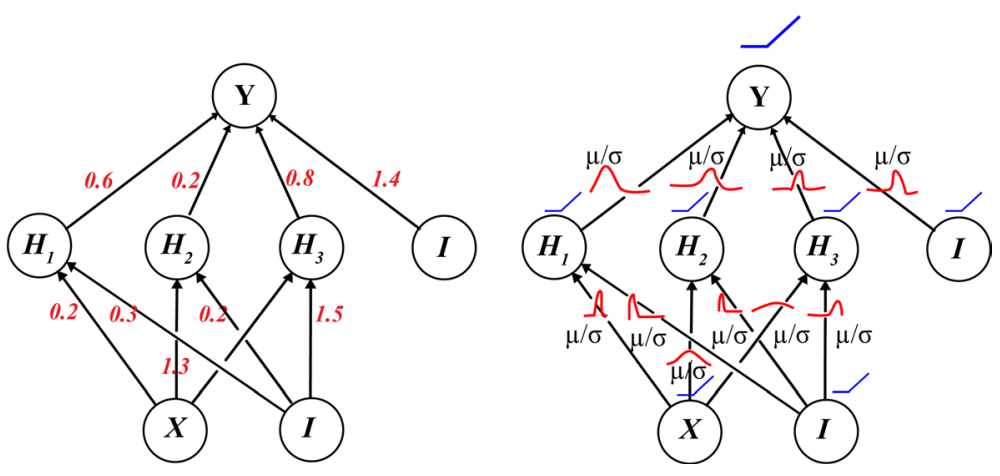

Fig. 5 Classical backpropagation sets fixed values as weights (left) while in Bayesian neural network a distribution is assigned to each weight (right)

Kendall et al. (2017) focused on epistemic uncertainty in computer vision using Bayesian deep learning models. They combined aleatoric uncertainty with epistemic uncertainty. The framework has been modelled with semantic segmentation and depth regression tasks. In addition, the formulated uncertainty resulted in a new loss function. This new loss function was robust to data uncertainty. In (Chen et al., 2013), a model has been created by a hierarchical convolutional factor-analysis. The parameters of layer-dependent model have been obtained by variational Bayesian analysis and Gibbs sampler. An online edition of variational Bayesian was used to handle large-scale and streaming data. Using beta-Bernoulli distribution, they estimated number of basic functions at each layer which is used for image processing applications.

Instead of Gaussian process (GP) to model distributions over functions, Snoek et al. (2015) used neural networks and showed that the performance of their method could overtake state-of-the-art GP-based approaches. However, unlike GP which scales cubically, this method scales linearly with the number of data. Using this modification, they have achieved intractable degree of parallelism, rapidly finding other models using convolutional networks, and some other applications such as image caption generation. Nowadays, one of the states-of-the-art fields is object detection using a deep convolutional neural network (CNN). Using this method, Zhang et al. (2015) extracted discriminative features for categorization. They used for localization using a Bayesian optimization to detect the object bounding box. In (Gal \& Ghahramani, 2015), as labelled data is hard to prepare, the authors have applied CNN on small data and hence faced the overfitting problem. To overcome this problem, Bayesian CNN is proposed which offered better robustness for small data. This has been obtained by pacing the probability distribution on CNN kernels. Bernoulli variational distributions are used to approximate the created model. Hence, they did not need additional model parameters. Theoretically, they cast dropout network training as approximate inference in the neural network. So, they used existing tools in deep learning without increasing the time complexity. Hence, achieved considerable improvement in accuracy as compared to other prevailing techniques.

Bayesian parameter estimation for the deep neural networks is suboptimal for issues with small datasets. This problem also exists where accurate posterior predictive densities are needed. Monte Carlo method can be utilized as one of the solutions for this problem. However, this method need to keep many copies for various parameters. It also need to make a prediction in many versions of the model. These two problems 
uses memory and time respectively. In the proposed method in (Korattikara et al. 2015), a more compact form of Monte Carlo approximation to the posterior predictive density is suggested. Then it is compared with two prevailing approaches: an approach depended on expectation propagation and variational Bayes. Authors claimed that, their method performed better than other two approaches.

Authors in (Dahl et al., 2013), used their proposed method with ReLUs and enhanced their overall system's performance by $4.2 \%$ with respect to deep neural networks (DNN) model trained with sigmoid units and 14.4\% with respect to the wellbuilt Gaussian mixture / hidden Markov technique. Also, this method needs minimum hyper-parameter tuning using a regular Bayesian optimization code.

In (Louizos et al., 2017), Bayesian method has been used to handle this problem. Two novelties of the proposed method are: (i) pruned nodes instead of individual weights and (ii) used posterior uncertainty to resolve the best possible fixed-point precision to decide the weights. These two factors improved the performance of the system. One of the flexible probabilistic models is Bayesian neural network with latent variables. These models can be used to estimate the network weights. Using these models, Depeweget al. (2018) showed the performance of decomposition of uncertainty for decision-making purposes. These methods can identify informative points of functions when these points had heteroscedastic and bimodal noise. By decomposition, they also define a risk-sensitive criterion for reinforcement learning. Using this learning method, a policy that balanced the expected cost, model-bias, and noise aversion can be found.

\subsection{Fuzzy logic}

Mathematicians define a fuzzy set as a class of objects whose elements have a degree of membership which is determined by a membership function. Fuzzy logic (Zadeh, 1988) is a method that its concepts are defined based on fuzzy set. In fuzzy logic, descriptive expressions are used for facts and rules expressions. The fuzzification operator is used to convert crisp values into fuzzy membership functions (Zadeh, 1988). Defuzzifying operations are used to map fuzzy membership functions into standard numbers which are used for decision and control purpose. To convert a standard system to fuzzy system, the following three steps are done (Zadeh, 1988).

(1) Convert the inputs into fuzzy membership functions (Fuzzification).

(2) Apply fuzzy rules to fuzzy input values to estimate the fuzzy outputs.

(3) Convert the fuzzy outputs to standard outputs (Defuzzification).

Fuzzy logic maybe used to model uncertainty (Dervishi, 2017; Khodabakhshi \& Moradi, 2017; Majeed Alneamy et al., 2019; Ornelas-Vences et al., 2017; Rundo, 2020; Sengur, 2008; Toğaçar et al. 2020). However, there is a more powerful extension of it dubbed adaptive neuro-fuzzy inference system (ANFIS) that integrated the learning capability of fuzzy logic with neural networks to model uncertainty in expressiveness. Fuzzy logic is utilized to model uncertain scenarios and that model is learned by neural network. In the next section, it will be explained in detail. 


\subsubsection{ANFIS}

The building block of ANFIS is Takagi-Sugeno fuzzy inference system (Karaboga \& Kaya, 2019). It is capable of capturing the benefit of fuzzy logic and artificial neural network both as it integrates two of them into a single framework. The IF-THEN fuzzy rules are utilized to learn approximate nonlinear functions in this inference system. The best parameters extracted from the genetic algorithm may be used to apply ANFIS in an optimal and efficient way. It is termed as a universal estimator and applies a situational aware intelligent energy management system. This architecture is comprised of five layers. The fuzzification and rule layers are the first and second layers respectively. The fourth layer takes the input from the third layer that normalized the values. The defuzzificated values are passed to the last layer that returns the final output.

The rule base comprises of a fuzzy if-then rules of Takagi and Sugeno's type as depicted below:

If $x$ is $A$ and $y$ is $B$ then $z$ is $f(x, y)$.

where $z=f(x, y)$ is a crisp function in the consequent, and A and B are the fuzzy sets in the antecedents. A first order Sugeno fuzzy model is formed if $f(x, y)$ is considered as first order polynomial. To form a first order two rule Sugeno fuzzy inference system, two rules may termed as below:

Rule 1: If $x$ is $A_{1}$ and $y$ is $B_{1}$ then $f 1=p_{1} x+q_{1} y+r_{1}$.

Rule 2: If $x$ is $A_{2}$ and $y$ is $B_{2}$ then $f_{2}=p_{2} x+q_{2} y+r_{2}$.

The type-3 fuzzy inference system as proposed by Takagi and Sugeno is used here. Its structure is shown in Fig. 6 (Liu, 2019).

In this inference system, the input variables added by a constant term linearly form the output of each rule.

The individual layers of ANFIS structure are described below: Layer 1: All the node $\mathrm{i}$ in layer 1 are adaptive with a node function

$$
O_{i}^{1}=\mu_{A_{i}}(x)
$$

where, $\mathrm{x}$ is the input to node $\mathrm{i}, \mu_{A_{i}}$ is the membership function of $\mathrm{A}_{\mathrm{i}}$ and $\mathrm{A}_{\mathrm{i}}$ is the linguistic variable related with this node function. The membership function is selected as below:

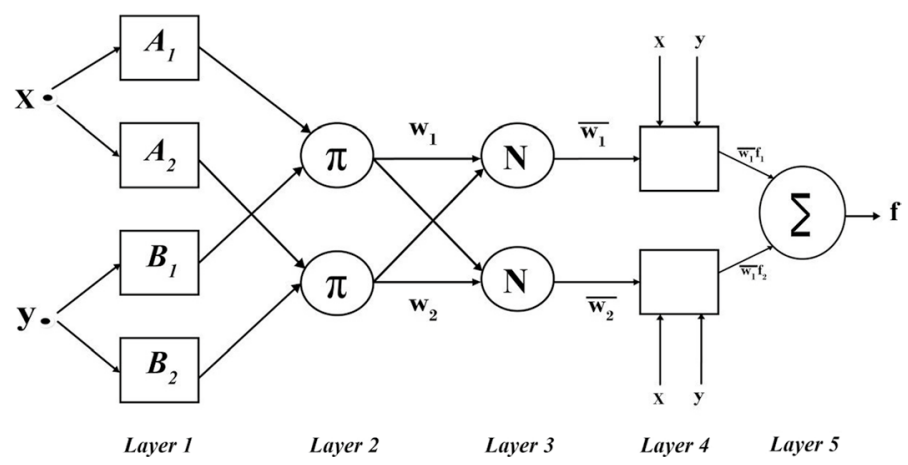

Fig. 6 Type-3 ANFIS structure 


$$
\mu_{A_{i}}(x)=\frac{1}{1+\left[\left(\frac{x-c_{i}}{a_{i}}\right)^{2}\right]^{b_{i}}}
$$

or

$$
\mu_{A_{i}}(x)=\exp \left\{-\left(\frac{x-c_{i}}{a_{i}}\right)^{2}\right\}
$$

where $\left\{\mathrm{a}_{\mathrm{i}}, \mathrm{b}_{\mathrm{i}}, \mathrm{c}_{\mathrm{i}}\right\}$ is the principle parameter set and $\mathrm{x}$ is the input.

Layer 2: The firing strength $\omega_{i}$ of a rule is measured by a fixed node that belongs to this layer. The product of each incoming signals to it is the output of this node and is depicted as below:

$$
O_{i}^{2}=\omega_{i}=\mu_{A_{i}}(x) \times \mu_{B_{i}}(y), i=1,2
$$

Layer 3: Layer 3 is represented as below:

$$
O_{i}^{3}=\overline{\omega_{i}}=\frac{\omega_{i}}{\omega_{1}+\omega_{2}}, i=1,2
$$

Layer 4: Every node in this layer is adaptive layer with node function described as below:

$$
O_{i}^{4}=\bar{\omega}_{i} f_{i}=\overline{\omega_{i}}\left(p_{i} x+q_{i} y+r_{i}\right), i=1,2
$$

where $\overline{\omega_{i}}$ is the output of the layer 3 and $\left\{\mathrm{p}_{\mathrm{i}}, \mathrm{q}_{\mathrm{i}}, \mathrm{r}_{\mathrm{i}}\right\}$ is the consequent parameter set.

Layer 5: It is consists of only one fixed node that measures the overall output as the summation of all incoming signals. It is described as below:

$$
O_{i}^{5}=\sum_{i} \bar{\omega}_{i} f_{i}=\frac{\sum_{i} \omega_{i} f_{i}}{\sum_{i} \omega_{i}}
$$

As shown in Fig. 6, the first and fourth layers are adaptive layers. Three adjustable parameters $\left\{\mathrm{a}_{\mathrm{i}}, \mathrm{b}_{\mathrm{i}}, \mathrm{c}_{\mathrm{i}}\right\}$ exist and associated with the input membership functions in the first layer. These parameters are dubbed as premise parameters. Three adjustable parameters $\left\{\mathrm{p}_{\mathrm{i}}, \mathrm{q}_{\mathrm{i}}, \mathrm{r}_{\mathrm{i}}\right\}$ exist in the fourth layer. These are termed as consequent parameters.

\subsubsection{Related works based on ANFIS}

The authors of (Turabieh et al., 2019) proposed a Dynamic ANFIS (D-ANFIS) to handle the missing values in the application used for the internet of medical things. This way they can overcome the potential problems that may occur. In (Ziasabounchi \& Askerzade, 2014), authors devised an ANFIS-based classifier to detect the degree of heart disease based on characteristic data of patients. The prediction model utilized seven variables as input. Their empirical results yielded an accuracy of $92.3 \%$ with k-fold cross-validation strategy.

Early diagnosis of chronic kidney disease (CKD) can prevent or reduce the progression of renal failure. Yadollahpour et al. (2018) proposed an ANFIS-based expert medical decision support system (MDSS) to predict the timeframe of renal damage. They considered the 
glomerular filtration rate (GFR) as the biological marker of renal failure. The ANFIS model utilized current GFR and diabetes mellitus as underlying disease, diastolic blood pressure, and weight as the effective factors in renal failure prediction. Their model predicted accurately the GFR variations using long future timeframes.

The authors of (Salah et al., 2013) designed a helping device for elderly people. The device is called E-JUST assistive device (EJAD). Inertial sensors and a motion capture system recognized human posture. The EJAD comprises of an active walker and a robot arm. Fuzzy system applying ANFIS is trained by the modified IMUs to the right posture of the patient.

One of the autoimmune ailments is rheumatoid arthritis (RA) that directs to significant mortality and morbidity. Özkan et al. (2010) extracted the key features from the left and right hand Ulnar artery Doppler (UAD) signals for the detection of rheumatoid arthritis disease by using multiple signal classification (MUSIC) techniques. ANFIS used the features derived from left and right hand UAD signals for the classification of RA. The hybrid model comprised of ANFIS and MUSIC techniques demonstrated accuracies of $91.25 \%$ using left hand UAD signals and $95 \%$ using the right hand UAD in the early recognition of rheumatoid arthritis disease.

In (Yang et al., 2014), the authors devised a risk assessment prediction model for coronary heart disease by optimizing linear discriminant analysis (LDA) and ANFIS methods. They applied a Korean Survey dataset. Their technique yielded a lofty prediction rate of $80.2 \%$ in preventing coronary heart disease.

The authors of (Polat \& Güneş, 2006) tried to use k-nearest neighbor (k-NN) and principal component analysis (PCA) based weighted for pre-processing of data, and ANFIS to diagnose thyroid disease. As the first step, they tried to reduce the dimension of thyroid disease data from five features to only two features using PCA. Then based on k-NN, a pre-processing phase was applied on data and finally, ANFIS was used for diagnosing thyroid disease.

In (Kumar et al., 2003), a recursive method has been used for the fuzzy system online learning employing Tikhonov regularization. This system was based on the recursive solution of a nonlinear least-squares problem. In (Ghazavi \& Liao, 2008), a collection of fuzzy systems was used. A fuzzy k-NN algorithm, fuzzy clustering, and ANFIS were applied on medical datasets. The results showed that feature selection is an important phase for time reduction and accuracy increasing of the proposed algorithm.

Papageorgiou (Papageorgiou, 2011) proposed the Fuzzy inference map to handle the problem of risk analysis and assessment of pulmonary infections in the hospital. Their proposed method is a soft computing algorithm which could deal with situations such as uncertain descriptions. In (Nguyen et al., 2015), a new method which is a combination of wavelet transformation (WT) and interval type-2 fuzzy logic system (IT2FLS) is used for medical noisy and high-dimensional data classification. IT2FLS could handle uncertainty and noise in complex medical data. Their method could serve as a decision support system a technique that is applied to compute the uncertainty in variety of fields such as financial problems and project management. Overall, this technique is used for predicting models and estimate the probability of a system outcome. It is used when we faced in clinical settings. A brief description of these works are summarized in Table 3.

\subsection{Monte Carlo simulation}

It is with a system having random variables. Monte Carlo (MC) simulation helps to predict all potential results of a system. This way, the users can take better decisions according to the risk and uncertainty of the system. This is why this method is referred 


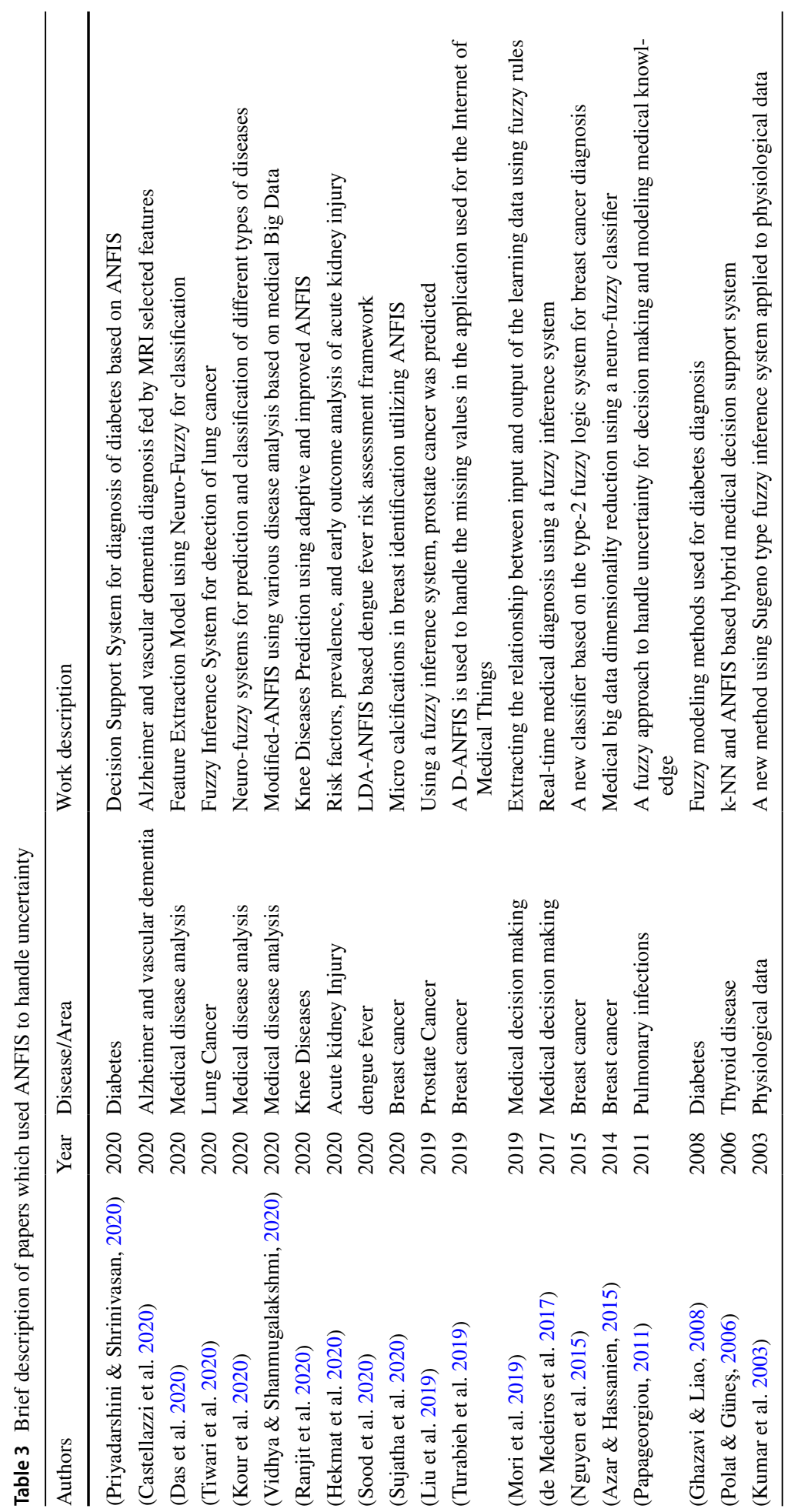


to as a probability simulation by some researchers (Arnaud Doucet et al., 2001). The main idea is that one can solve the problem using randomness. This method is commonly used in physical and mathematical problems when other methods are hard to be used. They are usually used for optimization and numerical integration (GrzymalaBusse, 1988).

\subsubsection{Monte Carlo method}

MC techniques are a subset of computational algorithms that utilize the procedure of repeated random sampling to obtain numerical estimations of unknown parameters. They assess the impact of risk and allow the modelling of critical situations where many random variables are engaged (Ghobadi et al., 2020; Hecquet et al., 2007; Jeeva \& Singh, 2015; Precharattana et al., 2011). The uses of the method are exceptionally widespread and have led numerous innovative discoveries in the fields of finance, game theory, and physics (Liesenfeld \& Richard, 2001). A broad range of Monte Carlo methods shares the generality that they depend on random number generation to crack deterministic problems (Koistinen, 2010).

\section{Basic principle Monte Carlo Integration}

Let us say $f$ is a density that we simulate from and are interested in the expectation (Liesenfeld \& Richard, 2001)

$$
I=\int h(x) f(x) d x=E h(X)
$$

Suppose from set $\mathrm{Y}_{\mathrm{i}}=\mathrm{h}\left(\mathrm{X}_{\mathrm{i}}\right)$ and density $\mathrm{f}$, we simulate $\mathrm{X}_{1}, \mathrm{X}_{2}, \ldots$ and then the sequence $\mathrm{Y}_{1}, \mathrm{Y}_{2}, \ldots$ is i.i.d. and $\mathrm{EY}_{\mathrm{i}}=\mathrm{Eh}\left(\mathrm{X}_{\mathrm{i}}\right)=\int h(x) f(x) d x=I$. We compute the $\mathrm{N}$ values $\mathrm{h}\left(\mathrm{X}_{1}\right), \ldots$, $\mathrm{h}\left(\mathrm{X}_{\mathrm{N}}\right)$ to acquire the estimate

$$
\hat{I}_{N}=\frac{1}{N} \sum_{i=1}^{N} h\left(X_{i}\right)
$$

By the SLLN, as N increases, $\hat{I}_{N}$ converges to I with the condition $E|h(x)|<\infty$. We are free to select $\mathrm{N}$ as large as available computer time in Monte Carlo simulations. It is easy to select the standard deviation and variance of the estimator. If the variance of the average is denoted as below then the variance of the single term $\mathrm{h}(\mathrm{X})$ is finite (Liesenfeld \& Richard, 2001).

$$
\operatorname{var} \hat{I}_{N}=\frac{1}{N} \operatorname{varh}(X)
$$

This is termed as Monte Carlo variance, simulation variance, or sampling variance of

the estimator $\hat{I}_{N}$. The accuracy of the $\hat{I}_{N}$ can be measured more meaningfully by applying the square root of the variance. The square root of the variance of an estimator is also dubbed as standard error. The standard error of a Monte Carlo estimate is termed as Monte Carlo the standard error, simulation standard error, or sampling standard error. The Monte Carlo standard error is of the order $1 / \sqrt{N}$, since 


$$
\sqrt{\operatorname{var} \hat{I}_{N}}=\frac{1}{\sqrt{N}} \sqrt{\operatorname{varh}(X)}
$$

The population variance or theoretical variance $\operatorname{var} h(X)$ that is required in both Eqs. 16 and 17 is generally unknown. It can be however measured by the sample variance of $h\left(X_{\mathrm{i}}\right)$ values,

$$
s^{2}=\widehat{\operatorname{var}} h(X)=\frac{1}{N-1} \sum_{i=1}^{N}\left(h\left(X_{i}\right)-\hat{I}_{N}\right)^{2}
$$

We achieve an approximate $100(1-\alpha) \%$ confidence interval for I namely

$$
\hat{I}_{N} \pm z_{1-\frac{\alpha}{2}} \frac{s}{\sqrt{N}}
$$

\subsection{Related works based on Monte Carlo Simulation (MCS)}

In (Papadimitroulas et al. 2012), extensive validation of MCS toolkit named as GATE is used to estimate dose point kernels (DPKs). It is widely used for many medical physics applications such as patient dosimetry and Computed Tomography (CT) image simulation. The results are compared with reference data to produce a total DPKs complete dataset for radionuclides in nuclear medicine.

In (Downes et al., 2009), the X-ray volume imager is modelled utilizing a novel Monte Carlo (MC) code. In this work, a novel constituent module was devised to precisely mould the unit's bowtie filter. The results showed good agreement between measurement and MC.

In (Chen et al., 2009), a new method is proposed to investigate scatter in CT breast imaging. It is done by comparing the distribution of measured scatter to those simulated using the Monte Carlo simulation toolkit (named as Gate). The results of scatter measurements are compared to previous ones. It was observed that from a non-breast source, a significant scatter can arise. The validated Monte Carlo simulation toolkit was also used to describe the scatter in different X-ray settings as well as for various breast sizes.

Authors in (Bush et al., 2008) generated an automated system named VIMC-Arc based on MC. Their designed system requires minimal user input like patient ID, requested dose uncertainty, and required voxel size. This system can be a great platform for the analysis of dosimetric problems with varying degrees of tissue inhomogeneity.

Jia et al. (Jia et al., 2011) developed a MC dose calculation package that used graphics processing unit)GPU( capabilities. They named their package as gDPM v2.0. They could achieve high computational power using GPU architecture without decreasing the accuracy. They tested their system on both phantoms and realistic patients using central processing unit (CPU) and GPU simulations. There is no significant difference between the accuracy of these simulations. However, GPU simulations are much faster than CPUs.

A good review on the capability of GATE Monte Carlo simulation was done in (Sarrut, 2014) for dosimetry applications and radiation therapy. The GATE MC simulation platform works based on the GEANT4 toolkit. Many applications that used GATE for radiotherapy simulations were reviewed in this research. An important feature of the GATE which makes it easy to model both treatment and image acquisitions within the same system was also emphasized. 
In (Lee et al., 2012)introduced a database established by a complete organ-effective dose i.e. (33 organs and tissues) based on CT scanner MC simulation. The test cases ranged from new born to 15-year-old male and female. The achieved results are compared with three existing researches. This comparison showed that phantoms using realistic anatomy is essential for better accuracy in CT organ dosimetry.

A simple approach proposed in Wang and Leszczynski (2007) evaluated the size and shape of a linac's focal spot by comparing the profiles with the MC calculated ones from the profile of measured dose data. A brief description of these papers are summarized in Table 4.

\subsection{Rough set theory (RST)}

After fuzzy, probability and evidence theories, a novel mathematical tool called RST [63] to deal with uncertain and inconsistent knowledge has been proposed. The applications based on RST have increased in recent years as more researchers are attracted to this area (Chen, 2013; Stokić et al. 2010; Wang et al. 2010; Zhang et al. 2010). In the artificial intelligence domain, it is one of the hot topics which is originated from information model. The basic concept comprised of two stages. The first stage forms rules and concepts via classification of relational databases. The second stage is to mine knowledge through classification for approximation of the target and classification of the equivalence relation.

\subsubsection{Formal definition}

Related data reasoning or analysis of algorithms and approximation of sets are critical research problems in the rough sets domain. Some basic concepts are described in this section. Let us consider an information system $I$ represented as the 4-tuple (Zhang et al. 2016)

$$
I=\langle U, X, V, f\rangle, X=C \cup D,
$$

where subsets $\mathrm{D}$ and $\mathrm{C}$ are called decision attribute set and condition attribute set, $\mathrm{X}$ is a finite nonempty set of attributes, $\mathrm{U}$ is a finite non empty set of attributes. $V=U_{a \in X} V_{a}$, where $V_{a}$ is the value of attribute $a, \mathrm{f}: \mathrm{X}>\mathrm{V}$ is a description function and card $\left(V_{a}\right)>1$.

Definition 1 (Indiscernible relation). An indiscernible relation ind(B) on the universe $\mathrm{U}$ given a subset of attribute set $B \subseteq X$ is defined as.

$$
\operatorname{ind}(B)=\left\{(x, y) \mid(x, y) \in U^{2}, \quad \forall_{b \in B}(b(x)=b(y))\right\}
$$

The pair $\left(\mathrm{U},[x]_{\text {ind }(B)}\right)$ is termed as approximation space, equivalence class of an object $\mathrm{x}$ is defined by $[x]_{i n d(B)}$ or $[\mathrm{x}]$ if no confusion arises (Zhang et al. 2016).

Definition 2 (Upper and lower approximation sets). Let $I=\langle U, X, V, f\rangle$ be an information set for a subset $Z \subseteq U$, its upper and lower approximation sets are termed respectively by (Zhang et al. 2016).

$$
\begin{gathered}
\underline{\operatorname{apr}(Z)=\{x \in U \mid[x] \cap Z \neq \emptyset\}} \\
\overline{\operatorname{apr}}(Z)=\{x \in U \mid[x] \in Z\}
\end{gathered}
$$




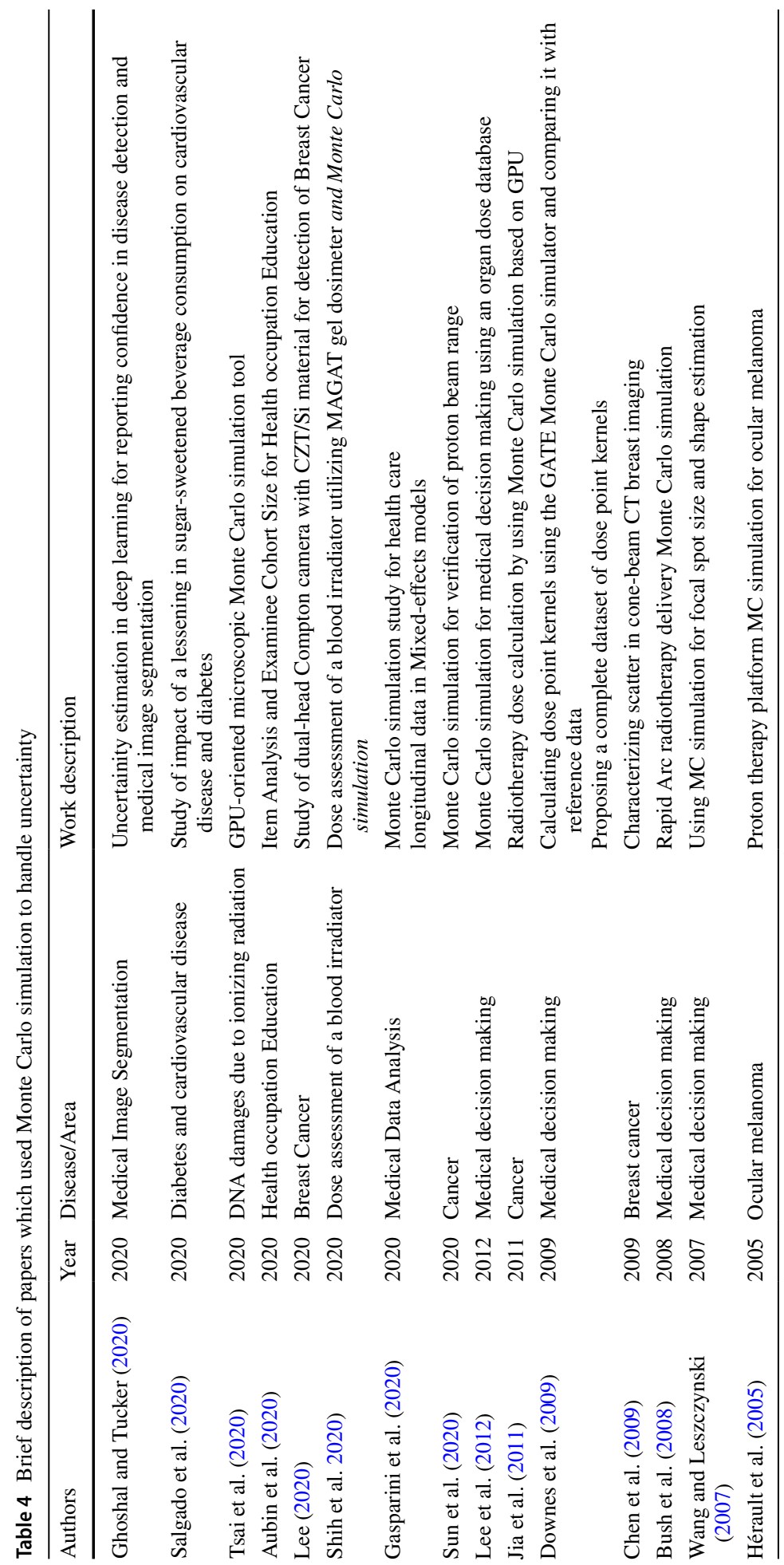


where $[\mathrm{x}]$ defines the equivalence class of $\mathrm{x}$.

Definition 3 (Definable sets) Let $I=\langle U, X, V, f\rangle$ be an information set for a target subset $Z \subseteq U$, and attribute subset $B \subseteq X$, if and only if $\overline{a p r}(\mathrm{Z})=\operatorname{apr}(\mathrm{Z}), \mathrm{Z}$ is termed as definable set with respect to $B$.

Definition 4 (Rough Sets) Let $I=\langle U, X, V, f\rangle$ be an information set for a target subset $Z \subseteq U$, and attribute subset $B \subseteq X$, if and only if $\overline{\operatorname{apr}}(\mathrm{Z}) \neq \operatorname{apr}(\mathrm{Z}), \mathrm{Z}$ is termed as rough set with respect to $\mathrm{B}$.

Definition 5 (Roughness of rough sets) Let $I=\langle U, X, V, f\rangle$ be an information set for a target subset $Z \subseteq U$, and attribute subset $B \subseteq X$, the roughness of set $Z$ is denoted as below with respect to $\mathrm{B}$,

$$
P_{B}(Z)=1-|\underline{\operatorname{apr}}(Z)| /|\overline{\operatorname{apr}}(Z)|
$$

where I.I defines the cardinality of a finite set and $Z \neq \emptyset$.

Three disjoint regions of a rough set are illustrated in Fig. 7 (Zhang et al. 2016). The boundary region leads to uncertainty in a rough set. The larger the boundary region will lead to the higher degree of uncertainty.

\subsection{Related works based on RST}

In (Kai-Quan, 2002), Shi discussed S-rough set and their application in diagnosis of diseases. The author also presented the characteristics and structures of S-rough set.

Jiang et al. (Jiang et al. 2017) proposed a new model for sub-health diagnosis with reference to traditional Chinese medicine (TCM). Fuzzy weighted matrixes are generated and sub-health classification is done after extracting key features by utilizing fuzzy mathematics and rough sets. The novel method proved its efficacy when compared to other models in this domain.

Fig. 7 Three disjoint regions of a rough set

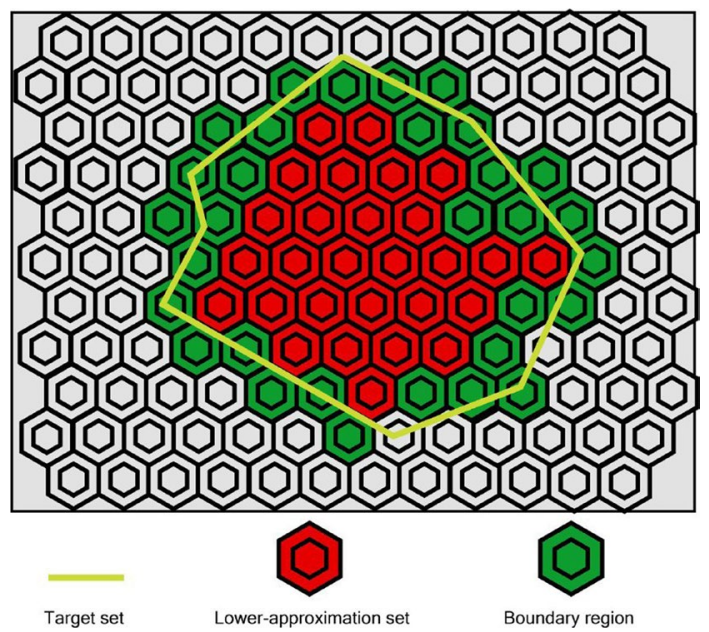


In (Ningler et al. 2009), a novel approach by slightly modifying the variable precision rough set model is presented. In this research, Ningler et al. tested both the models using the dataset containing electroencephalogram of anesthetized and awake patients. Their technique produced smaller rule sets and achieved better cutback of features for inconsistent or noisy records despite of higher computational effort.

Wang et al. (Wang et al. 2010) devised a novel tumor classification technique based on neighborhood rough set and an ensemble of probabilistic neural network model based gene reduction. Gene ranking is applied to select informative genes and minimum gene subsets are chosen by reducing gene. Their method recorded competitive performance and is not sensitive to initially selected genes.

In (Tsumoto, 1998a), authors proposed a rule induction technique which extracted classification rules as well as medical knowledge for the diagnosis of disease. The model is tested on three clinical datasets, whose results induced diagnostic rules correctly and estimated statistical measures as well.

Chou et al. (Chou et al. 2007) utilized rough set theory (RST) and self-organizing map (SOM) methods to evaluate laboratory test and drug utilization to extract knowledge from the raw data of cardiovascular disease patients. The model achieved an accuracy of $98 \%$ and detected the trend of patient's condition individually.

In (Tsumoto, 1998b), the characteristics of expert rules were examined and a new method to derive plausible rules by utilizing three procedures was proposed. The proposed technique was evaluated on medical datasets and experts' decision processes were represented by induced rules. A brief description of these papers are summarized in Table 5 .

\subsection{Dempster-Shafer theory (DST)}

P. Dempster and his student Glenn Shafer introduced Dempster Shafer Theory (Denźux, 2016). The theory tried to overcome the limitations of Bayesian methods. Bayesian probability cannot describe ignorance and Bayesian theory concerns about single evidences. It is an evidence theory and it integrates all possible outcomes of the problem. The uncertainty in this model is as follows:

1. All possible outcomes are considered.

2. Belief will direct to believe in some likelihood by carrying out some evidences.

3. Plausibility will make evidence compatibility with possible outcomes.

\subsubsection{Formal definition}

All possible states of a system taken into account by the set termed as Z, the universe.

The set of all subsets of $Z$ including empty set $\varnothing$ is represented by the power set $2 Z$.

Suppose, if $Z=\{a, b\}$, then.

$$
2^{z}=\{\phi,\{a\},\{b\}, Z\}
$$

Propositions are represented by the elements of the power set concerning the actual state of the system, by having all and only the state in which the proposition is true. A belief mass to each element is assigned by the theory of evidence. Formally, a function $\mathrm{m}$ : $2^{\mathrm{Z}} \rightarrow[0,1]$ is termed as basic belief assignment, having two properties. First property is "the mass of the empty set is zero" i.e. $\mathrm{M}(\varnothing)=0$.

Secondly, the masses of the remaining members of the power set added up to total of 1 . 


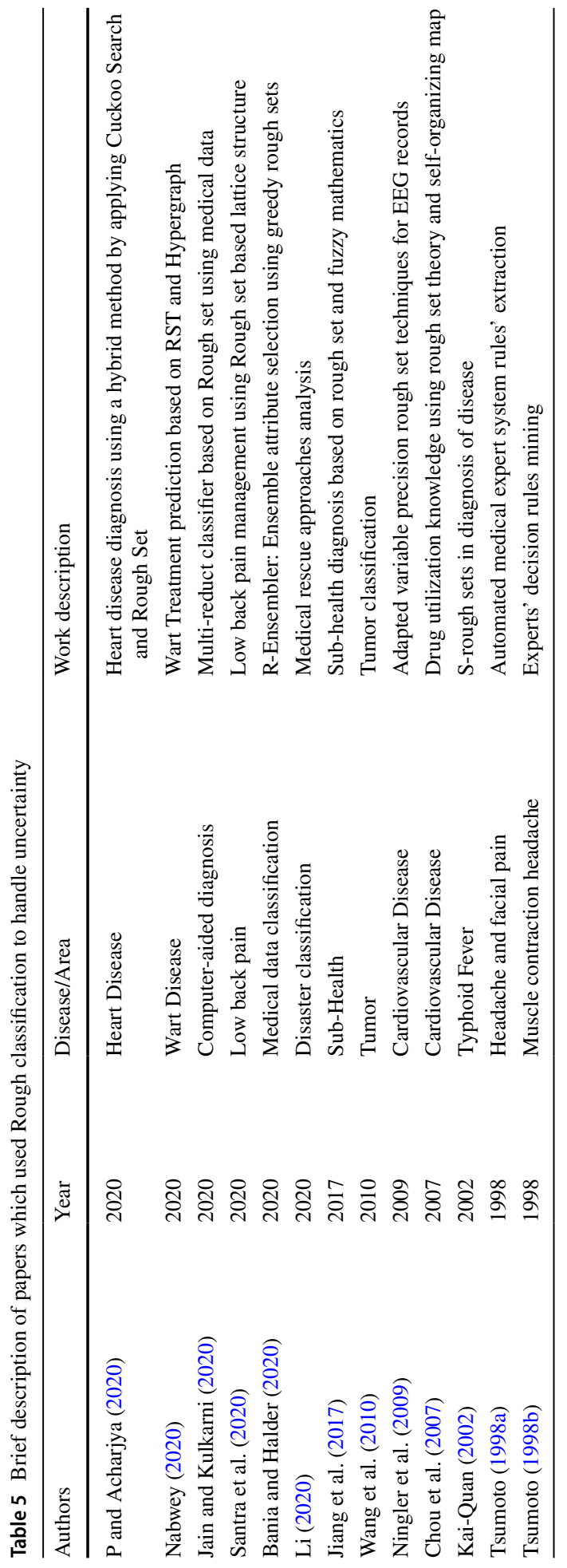




$$
\sum_{L \in 2^{Z}} m(L)=1
$$

The upper and lower bounds of a probability interval can be described from the mass assignments. The interval comprised of the precise probability of a set and is bounded by two non-additive continuous measures called plausibility and belief.

$$
\operatorname{bel}(L) \leq P(L) \leq p l(L)
$$

The belief bel (L) for a set $\mathrm{L}$ is denoted as the addition of all the masses of subsets of the set of interest.

$$
\operatorname{bel}(L)=\sum_{N \mid N \subseteq L} m(N)
$$

The plausibility $\mathrm{pl}(\mathrm{L})$ is the addition of all the masses of the sets $\mathrm{N}$ that intersect the set of interest $\mathrm{L}$.

$$
p l(L)=\sum_{N \mid N \cap L \neq \emptyset} m(N)
$$

The plausibility and belief are related to each other as follows:

$$
\operatorname{pl}(L)=1-\operatorname{bel}(\bar{L})
$$

Conversely for finite set $\mathrm{L}$, we can denote the masses of $\mathrm{m}(\mathrm{L})$ with the following inverse function given the belief measure bel $(\mathrm{N})$ for all subsets $\mathrm{N}$ of $\mathrm{L}$.

$$
m(L)=\sum_{N \mid N \subseteq L}(-1)^{|L-N|} \operatorname{bel}(N)
$$

where the difference of the cardinalities of the two sets is represented by $\mid \mathrm{L}-\mathrm{NI}$.

\subsubsection{Dempster's rule of combination}

The two sets of masses $\mathrm{m}_{1}$ and $\mathrm{m}_{2}$ are combined to depict joint mass and represented as below:

$$
\begin{gathered}
m_{1,2}(\emptyset)=0 \\
m_{1,2}(L)=\left(m_{1} \oplus m_{2}\right)(L)=\frac{1}{1-K} \sum_{N \cap C=A \neq \emptyset} m_{1}(N) m_{2}(C)
\end{gathered}
$$

where

$$
k=\sum_{N \cap C=\emptyset} m_{1}(N) m_{2}(C)
$$

$\mathrm{k}$ is an estimate of the amount of conflict between the two mass sets.

The characteristics of DST has ignorance part such that probability of all events cumulative to 1. Ignorance is reduced in this theory by incorporating more and more evidences and combination rule. The rule is utilized to integrate various types of possibilities. This 
theory has much lower level of ignorance. Uncertainty interval can be reduced by adding more information. The disadvantage of this theory implies that if the computation is high, we have to deal with $2^{\mathrm{n}}$ of sets.

\subsubsection{Related works based on DST}

Fuzzy soft set-based decision making has improved the uncertainty especially in the medicine field by the authors in [72]. They utilized DST and ambiguity measures for this purpose. Their proposed approach proved to be efficient and feasible as it improved performance by reducing the uncertainty caused due to subjectivity.

Authors in (Porebski et al., 2018) proposed a diagnosis support method and a rule selection both by applying fuzzy set and Dempster-Shafer theories. They used their model to diagnose liver fibrosis. They extracted information from a real dataset containing hepatitis C patients.

Authors (Xiao, 2018) proposed a hybrid framework utilizing DST with belief entropy. The feasibility and efficacy of their method are validated by implementing a numerical example and a medical application.

Straszecka et al. (2006) introduced a unified fuzzy-probabilistic approach for medical diagnosis modelling processes. The basic concepts of DST, i.e. a basic probability assignment and focal elements correspond to the impact of an individual symptom in the diagnosis and disease symptoms, respectively. Focal elements' interpretation as fuzzy sets, evidence uncertainty, and imprecision of diagnosis are the novel approaches in their method.

Biswas et al. (2020) presented a novel decision-making strategy based on DSTby applying soft fuzzy sets for elucidation of pneumonia malformation in low-dose x-ray images.

In (Ghasemi et al., 2013), authors combined Dempster-Shafer Theory and fuzzy inference system for brain MRI segmentation where the spatial information and the pixel intensities were utilized as features. The novelty of their work is that rules are paraphrased as evidences. The experimental results demonstrated that the proposed method called fuzzy Depster-Shafer inference system (FDSIS) exhibited competitive output using both real and simulated MRI databases.

The study in Shi et al. (2018) integrated local classification model (LCM) to predict the drug-drug interactions via DST. Their supervised fusion rule combined the results from multiple LCMs. Their LCM-DS model exhibited better performance as compared to three different prevailing approaches.

Authors in Kang et al. (2018) proposed a prognostic model based on DST and Gaussian mixture model for Clostridium difficile contagion. Criteria ratings of risk factors generated by the model helped the hospital administrators and risk managers to control and predict of Clostridium difficile infection prevalence.

Li et al. (2015) presented a framework based on fuzzy soft set and DST to help the clinicians in medical diagnosis. It has proved to be effective and feasible application in medical diagnosis.

Researcher in Bloch (1996) exploited crucial features of DST in medical imaging. He pointed out the key aspects of the theory. It included the introduction of global or partial ignorance, the computation of conflict between images, and modelization of both imprecision and uncertainty in medical image processing. Partial volume effect in MR images can be managed properly by this approach.

Wang et al. (2015) extended fuzzy DST to model domain knowledge using fuzzy and probabilistic uncertainty for medical diagnosis. A novel evidential structure to mitigate 
information loss is proposed. They presented novel intuitionistic fuzzy evidential reasoning (IFER) methodology that integrated inclusion measure and intuitionistic trapezoidal fuzzy numbers to enhance the accuracy of reasoning and representation.

Raza et al. (2006) utilized DST to fuse the results of classification of breast cancer data from two sources: Fine-Needle Aspirate Cytology and gene-expression patterns in peripheral blood cells data. The support vector machine with polynomial, linear, and Radial Base Function kernels are used for classification. The output of the fused classifiers yielded better results in detecting the breast cancer automatically.A brief summary of these works are summarized in Table 6.

\subsection{Imprecise probability}

Imprecise probability can be achieved by generalizing traditional probability. A set of probabilities with lower and upper probabilities $\operatorname{Prob}(Z)=\left[p_{1}, p_{2}\right]$ are applied instead of using probabilistic measure $\operatorname{Prob}(Z)=p$ related with an event $\mathrm{Z}$ for quantification both epistemic and aleatory uncertainties [84]. Several representations and theories of imprecise probability have been devised such as the coherent lower prevision theory, possibility theory, de Finetti's subjective probability theory, and Dempster-Shafer evidence theory.

\subsubsection{Definitions}

Consider upper probability $\bar{P}(\underline{\mathrm{Z}})$ and lower probability $\underline{P}(\mathrm{Z})$, with $0 \leq \underline{P}(\mathrm{Z}) \leq \bar{P}(\mathrm{Z}) \leq 1$. There is precise probability if $\bar{P}(\mathrm{Z})=P(\mathrm{Z})=\mathrm{P}(\mathrm{Z})$ for all events A. There is lack of knowledge completely about $\mathrm{Z}$ if $\bar{P}(\mathrm{Z})=1$ and $\underline{P}(\mathrm{Z})=0$. For disjoint events $\mathrm{Z}$ and $\mathrm{Y}$ :

$$
\begin{gathered}
\underline{P}(Z \cup Y) \geq \underline{P}(Z)+\underline{P}(Y) \text { and } \bar{P}(Z \cup Y) \leq \bar{P}(Z)+P(Y) \\
\underline{P}(\text { not }-Z)=1-\bar{P}(Z)
\end{gathered}
$$

Precise probability distributions of closed convex set $\mathcal{P}$ :

$$
\underline{P}(Z)=\inf _{p \in \mathcal{P}} p(Z) \operatorname{and} \bar{P}(Z)=\sup _{p \in \mathcal{P}} p(Z)
$$

Subjective interpretation:

$\bar{P}(\mathrm{Z})$ : minimum cost at which selling the gamble is desirable.

$\underline{P}(\mathrm{Z})$ : maximum cost at which buying gamble paying 1 if $\mathrm{Z}$ occurs and else 0 is desirable.

$\underline{P}(\mathrm{Z})$ can be deduced as reflecting the evidence in favor of event $\mathrm{Z}, 1-\bar{P}(\mathrm{Z})$ as reflecting the evidence against $\mathrm{Z}$, in favor of not- $Z$. of Z.

Imprecision $\Delta(\mathrm{Z})=\bar{P}(\mathrm{Z})-\underline{P}(\mathrm{Z})$ reflects lack of perfect information about probability

\subsubsection{Related works on imprecise probability}

Coletti et al. (2000) presented the role of coherence in handling and eliciting imprecise probabilities and its application to medical analytics. They also focused on the distinction between syntactic and semantic aspects. Mahmoud et al. (2016) used different machine learning decision tree classification algorithms on noisy medical datasets. They employed 


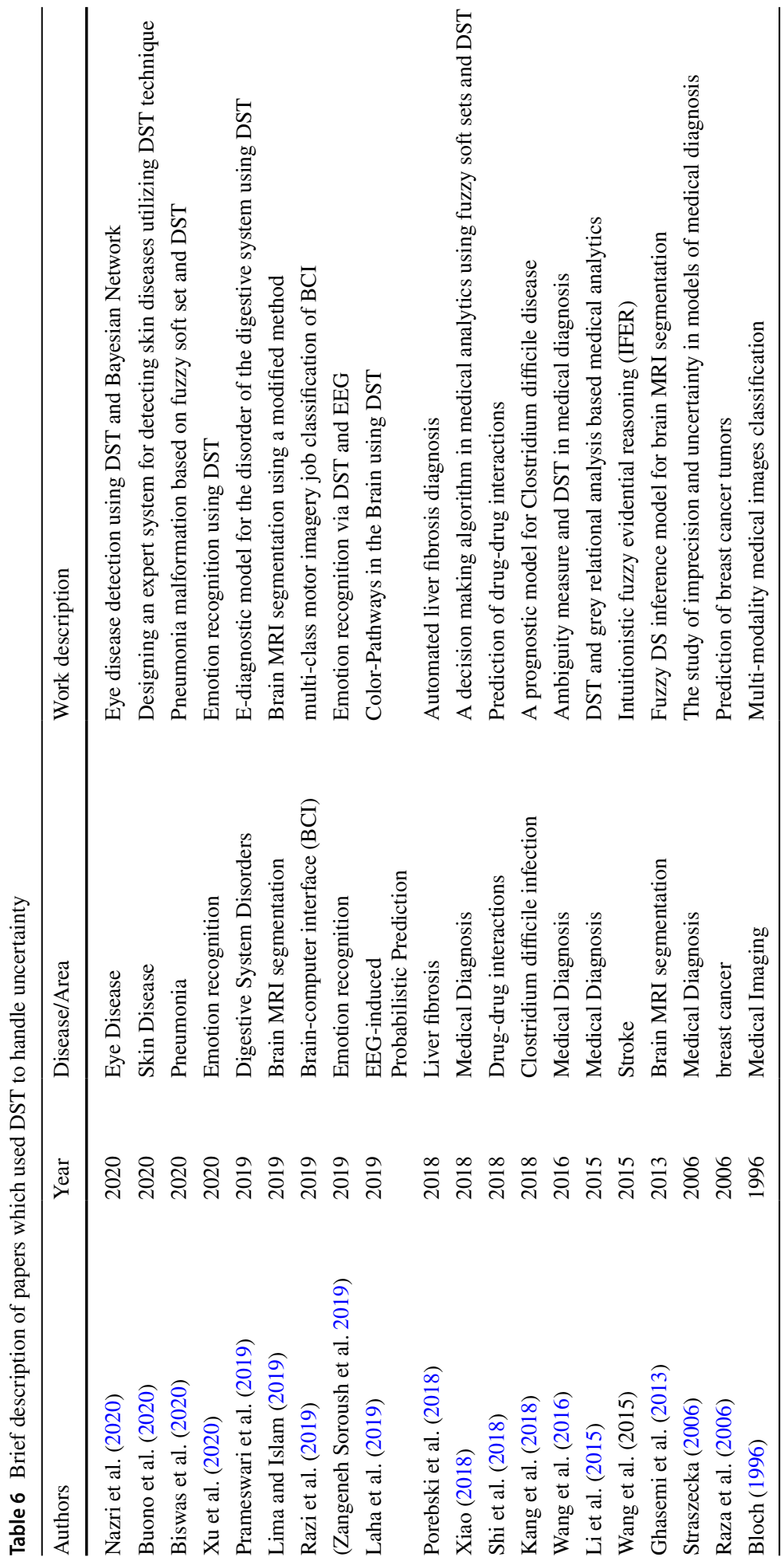


three decision tree methods: single tree classifiers, ensemble models, and creedal decision trees (CDTs) to tackle the uncertainty measures and imprecise probabilities. CDTs outperformed other two methods in noisy environments. Van Wyk et al. (2020) investigated the medicinal plants in African region. They studied traditional diversity patterns in selection of medicinal plants of Africa. They utilized imprecise Dirichlet model with linear regression and Bayesian analysis.

Kwiatkowska et al. (2009) revisited the concept of uncertainty, incompleteness, and imperfection of data. They studied the traditional hierarchical approach to knowledge, information, and data with reference to medical data, which is characterized by timedependency, variable granularity and heterogeneity. They argued that it is contextual to interpret the imprecision and medical data could not be decoupled from their intended usage. They integrated multidimensional, fuzzy-logic, and semiotic approaches to propose a framework for medical data modelling to address contextual interpretation of imprecision issues. A brief description of these papers are summarized in Table 7.

\section{Discussion}

Studying uncertainty involves uncertainty in data and uncertainty in the model. Data uncertainty arises from sources such as measurement noise, transmission noise, and missing values. Model uncertainty comprises of not knowing the best architecture and parameters which can predict future data. Uncertainty quantification helps to enhance the confidence in the results obtained by different methods. Nowadays, the growth of new technologies has paved the way to produce huge amounts of raw data in different fields. The use of such raw data is never easy as it might include noise. There are few researches like (Oh et al. 2018) did not perform noise filtering because of end to end training of deep learning network using noisy ECG signals. We know that CNN is less sensitive to noise. It can extract information even when the data are noisy (Qian et al. 2016). In another research (Karimifard \& Ahmadian, 2011), Hermitian basis functions are used to extract higher order cumulants from the ECG beats. They could reduce the effects of Gaussian noise. However, before getting useful information from data, they are cleaned for the presence of any noise and unnecessary information. Hence, dealing with uncertainty in both data and model is an important subject for researchers to make accurate decisions in various domains. Thus, researchers across the world are trying to deal with uncertainty using machine learning algorithms and probability theories. We have discussed algorithms namely Bayesian inference, fuzzy systems, Monte Carlo simulation, rough classification, Dempster-Shafer theory, and imprecise probability to handle the uncertainties. Figure 8 shows the percentage of uncertainty handling algorithms used in medical field by researches.

It can be noted from Fig. 8 that, Bayesian inference, fuzzy systems, and Monte Carlo simulation respectively have been used by many researchers in the medical field. Among these methods, Bayesian inference is more widely used as compared to other methods (Dempster, 1968; Ma et al. 2006; Minka, 2001). The involvement of average of parameters makes this method self-regularized. Both uncertainty types are tackled by this method. Prior knowledge is included while using Bayesian inference. But it suffers from few disadvantages such as it is computationally intensive. The intractability of its integrals and high dimension are other disadvantages of this method. Fuzzy systems are the second most widely used technique. These methods are simple and also can overcome different types of uncertainty efficiently (Kosko, 1994). MCS has few advantages such as it can address 


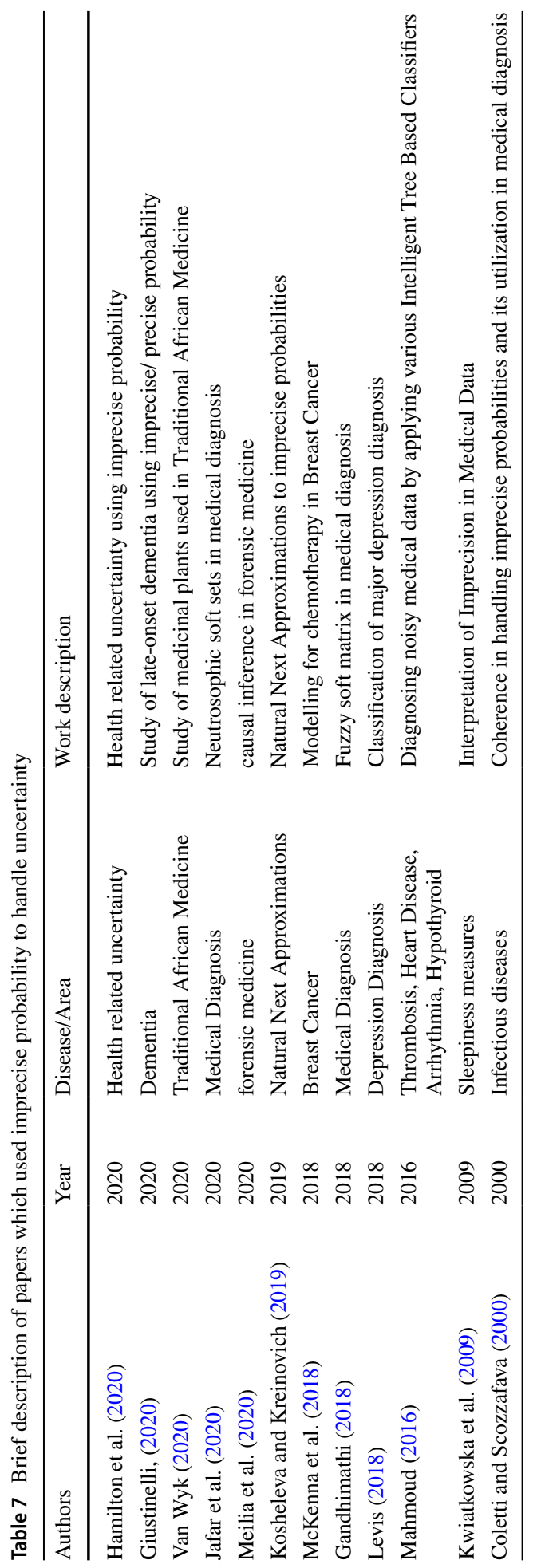


Fig. 8 Percentage of uncertainty handling algorithms used in medical field researches

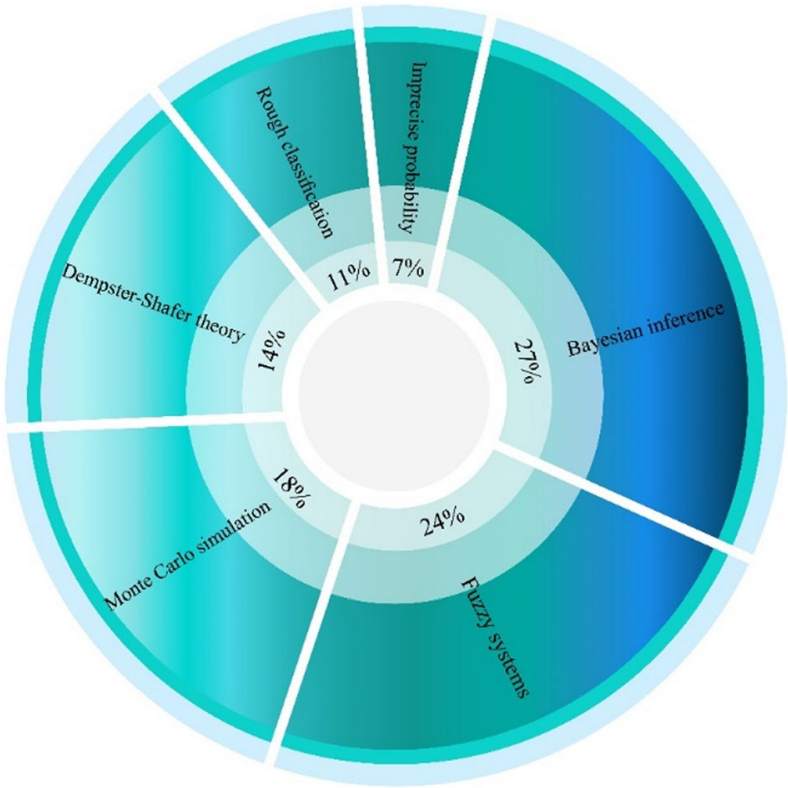

the intractability analytically and can survey the parameter space of a problem completely. Meanwhile, the MCS results are relatively easy to understand, flexible and empirical distributions can be handled. The limitation of MCS is its computational cost and solutions are not exact. They depend on the number of repeated runs (Mooney, 1997). Dempster-Shafer theory in combination with fuzzy systems also attracted the attention of many researchers (Wang et al. 2016). The main disadvantage of the rough set (Dash \& Patra, 2013) is that it cannot handle real-valued data. Using a real-valued rough set is the best alternative to solve this problem. Meanwhile, fuzzy-rough set theory can be used to handle this problem (Jensen \& Shen, 2004; Zhai, 2011). A brief description of the pros and cons of methods used are summarized in Table 8.

Few other methods can also be used to tackle the uncertainty quantification. Some of them are Bayesian information criterion (Pho et al. 2019), Laplace approximation (Friston et al. 2007), variational approximations (Ormerod \& Wand, 2010), exact sampling (Propp $\&$ Wilson, 1996), and expectation propagation (Minka, 2001). However, these methods are not much used by the researches.

There are also few challenges when investigating this topic. Few of them are: 1) handling dimensionality and computational cost of the problems, 2) testing the validity of different methods which can be used to handle uncertainty, 3) testing the validity of achieved results, 4) adaptation of model inputs to specific patient setting, and finally 5) determining the model complexity level which yielded more accurate outcomes.

In the fourth challenge, the inputs may include the computational domain, boundary conditions, and physical parameters. Measurement uncertainty and large biological variability lead to hampering of all measurements of these inputs. Consequently, it leads to uncertainties in the inputs. Meanwhile, some of the model inputs are immeasurable or they are measurable but costly.

The model complexity increases if it has to describe the reality more accurately is the fifth challenge. Consequently, it lessens the uncertainty in the output of the model. 


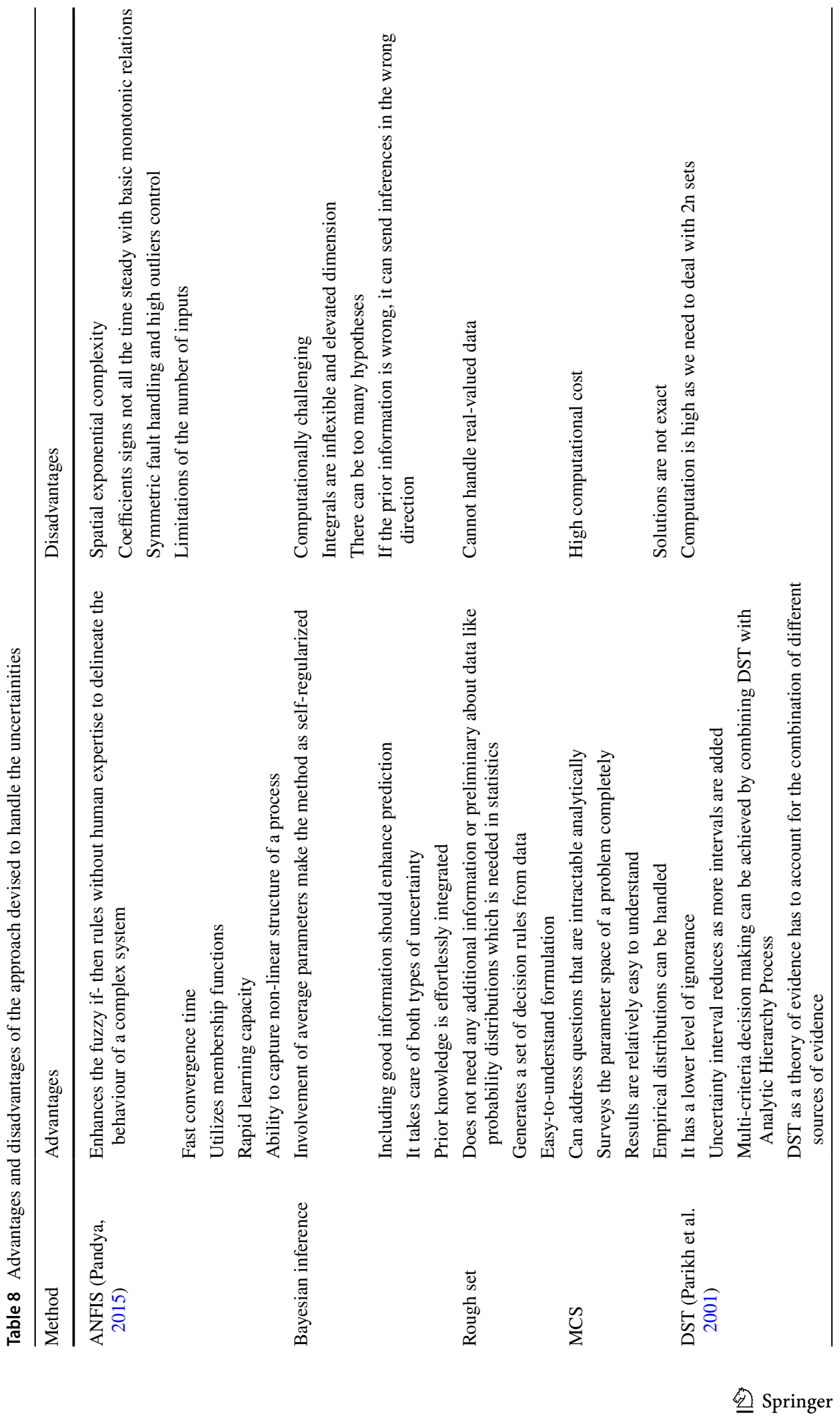




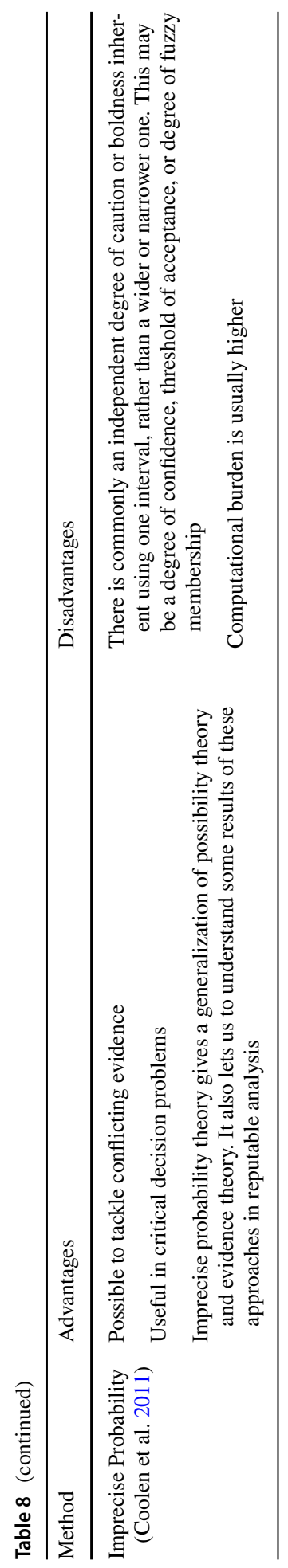

Springer 
However, this enhances the uncertainty in the input of the system simultaneously because more inputs of the system ought to be examined patient-specifically. As illustrated in Fig. 9, it is necessary to find an optimal transaction between the uncertainty resulting from system and data that can initiate nominal total uncertainty (Huberts et al. 2014). To deal with challenges number four and five, it is essential to measure the output uncertainty of the system. The uncertainties in the inputs of the system are responsible for it. The pros and cons of it developed to handle the uncertainties are summarized in Table 8.

Higher order statistics/spectra (HOS) (Chua et al. 2010) and deep learning (DL) techniques are found to more robust to noise. The bispectrum and cumulants widely used for medical data to capture the subtle changes in the signal (Pham, 2020). It can detect deviations from linearity, stationarity or Gaussianity in the signal. In nature, the biomedical signals are commonly non-stationary, non-linear and non-Gaussian. So, it is better to analyse them with HOS rather than the use of second-order correlations.

We know that data collection without any noise in the data is expensive and time-consuming. Nowadays, due to the progress in storage devices and technologies, a huge amount of medical data are available. These medical data are noisy and need to be eliminated to get an accurate diagnosis. HOS and deep learning techniques are found to be suitable to handle this type of noisy data. Deep neural networks can generalize on noisy data in training, instead of just memorizing them (Rolnick et al., 2017). In addition, it is shown in (Rolnick et al., 2017), deep neural networks can learn from data that has been impacted by an arbitrary amount of noise. By applying deep neural networks on multiple datasets such as MINST, CIFAR-10 and ImageNet, the authors showed that successful learning is possible even with an essentially arbitrary amount of noise. Authors have showed that using deep learning techniques, it is possible to get excellent performance even with noisy electrocardiogram (ECG) signals (Acharya et al., 2017; Gao et al., 2019; Jeong et al., 2019; Oh et al., $2018,2019)$. Hence, in future, DL models may be explored to nullify the presence of noise in the medical data.

\section{Conclusions and future works}

This paper reviewed uncertainty quantification challenges in the medicine/health domain. Unlike industrial fields, the medical field has always been determined by several factors leading to uncertainty in decisions and outcomes.

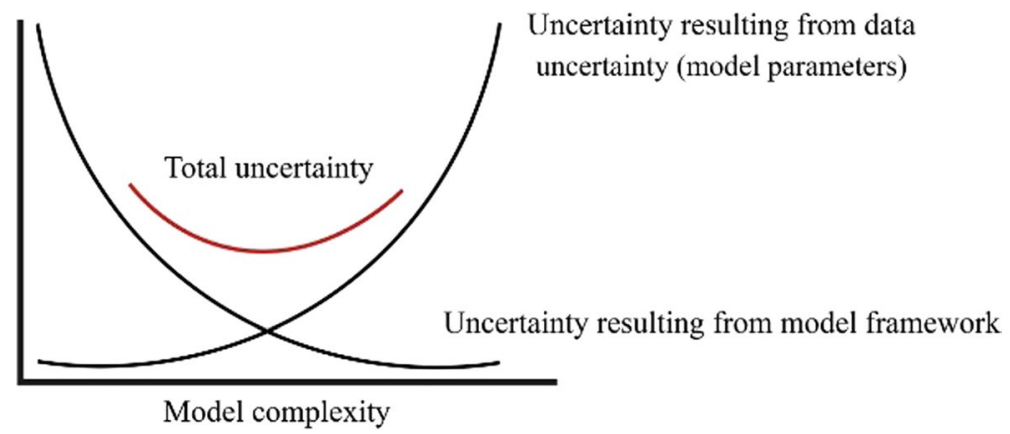

Fig. 9 The trade-off between data and model uncertainty 
More useful methods of handling uncertainty in healthcare should start with physicians. To achieve this goal, first, doctors need to have a clear and better picture of uncertainty in their work and strive for novel approaches to handle this challenge. Decision making in this field is intricate. So, it needs intuitive and rational thinking. Although uncertainty is an inevitable factor in medical decisions, clinicians commonly downplay its importance.

We know that artificial intelligence methods automate the processes and make them available more widely. However, it is not being used to its full potential in the medical field. Artificial Intelligence can be applied fully in medical domain once all aspects including uncertainty quantification are considered. With the help of artificial intelligence, early diagnosis of disease becomes a reality and also saves time and money.

The findings of this review paper demonstrated that different types of classical machine learning and probability theory techniques have been significantly used in handling the uncertainty in the data. Recently deep learning techniques are becoming very popular among researchers due to its high performance. Using deep learning methods high arrhythmia classification is obtained even with noisy data (Acharya et al. 2017; Oh et al. 2018, 2019). So, in future, such techniques can be employed to handle the uncertainty in the medical data and obtain high performance.

Funding The authors have not declared a specific grant for this research from any funding agency.

Data availability The authors declare that all data supporting the findings of this study are available within the paper.

\section{Declarations}

Conflicts of interest The authors declare that they have no conflict of interest.

\section{References}

Acharya, U. R., Fujita, H., Oh, S. L., Hagiwara, Y., Tan, J. H., \& Adam, M. (2017). Application of deep convolutional neural network for automated detection of myocardial infarction using ECG signals. Information Sciences, 415-416, 190-198.

Akkoyun, E., Kwon, S. T., Acar, A. C., Lee, W., \& Baek, S. (2020). Predicting abdominal aortic aneurysm growth using patient-oriented growth models with two-step Bayesian inference. Computers in Biology and Medicine, 117, 103620. https://doi.org/10.1016/j.compbiomed.2020.103620

Alizadehsani, R., et al. (2019a). Machine learning-based coronary artery disease diagnosis: A comprehensive review. Computers in Biology and Medicine, 111, 103346. https://doi.org/10.1016/j.compb iomed.2019.103346

Alizadehsani, R., et al. (2019b). A database for using machine learning and data mining techniques for coronary artery disease diagnosis. Scientific Data, 6, 227. https://doi.org/10.1038/s41597-019-0206-3

Alizadehsani, R., et al. (2019c). Model uncertainty quantification for diagnosis of each main coronary artery stenosis. Soft Computing:1-12

Alizadehsani, R., et al. (2020). Hybrid genetic-discretized algorithm to handle data uncertainty in diagnosing stenosis of coronary arteries expert systems

Alizadehsani, R., et al. (2021). Coronary artery disease detection using artificial intelligence techniques: a survey of trends, geographical differences and diagnostic features 1991-2020. Computers in Biology and Medicine, 128, 104095. https://doi.org/10.1016/j.compbiomed.2020.104095

Arabasadi, Z., Alizadehsani, R., Roshanzamir, M., Moosaei, H., \& Yarifard, A. A. (2017). Computer aided decision making for heart disease detection using hybrid neural network-Genetic algorithm. Computer Methods and Programs in Biomedicine, 141, 19-26. https://doi.org/10.1016/j.cmpb. 2017.01.004

Arnaud, D., de Freitas, N., Neil, G., (2001). An introduction to sequential Monte Carlo Methods. In Sequential Monte Carlo Methods in Practice. Springer, pp 3-14 
Asgharnezhad, H., Shamsi, A., Alizadehsani, R., Khosravi, A., Nahavandi, S., Sani, Z.A., Srinivasan, D. (2020), Objective Evaluation of Deep Uncertainty Predictions for COVID-19 Detection arXiv preprint arXiv:201211840

Ashby, D. (2006). Bayesian statistics in medicine: a 25 year review. Statistics in Medicine, 25, 35893631. https://doi.org/10.1002/sim.2672

Aubin, A.-S., Young, M., Eva, K., St-Onge, C. (2020), Examinee cohort size and item analysis guidelines for health professions education programs: A Monte Carlo simulation study academic medicine 95

Azar, A. T., \& Hassanien, A. E. (2015). Dimensionality reduction of medical big data using neural-fuzzy classifier. Soft Computing, 19, 1115-1127.

Bania, R. K., \& Halder, A. (2020). R-Ensembler: A greedy rough set based ensemble attribute selection algorithm with kNN imputation for classification of medical data. Computer Methods and Programs in Biomedicine, 184, 105122. https://doi.org/10.1016/j.cmpb.2019.105122

Biswas, B., Ghosh, S. K., Bhattacharyya, S., Platos, J., Snasel, V., \& Chakrabarti, A. (2020). Chest X-ray enhancement to interpret pneumonia malformation based on fuzzy soft set and DempsterShafer theory of evidence. Applied Soft Computing, 86, 105889.

Bloch, I. (1996). Some aspects of Dempster-Shafer evidence theory for classification of multi-modality medical images taking partial volume effect into account. Pattern Recognition Letters, 17, 905-919.

Buono, M. L. C., Pandiangan, N., \& Loppies, S. H. D. (2020). The Implementation Of An Expert System In Diagnosing Skin Diseases Using The Dempster-Shafer Method. Journal of Physics: Conference Series, 1569, 022028. https://doi.org/10.1088/1742-6596/1569/2/022028

Bush, K., Townson, R., \& Zavgorodni, S. (2008). Monte Carlo simulation of RapidArc radiotherapy delivery. Physics in Medicine and Biology, 53, N359-N370.

Castellazzi, G., et al. (2020). A machine learning approach for the differential diagnosis of Alzheimer and Vascular Dementia Fed by MRI selected features. Frontiers in Neuroinformatics. https://doi. org/10.3389/fninf.2020.00025

Chen, Y.-S. (2013). Modeling hybrid rough set-based classification procedures to identify hemodialysis adequacy for end-stage renal disease patients. Computers in Biology and Medicine, 43, 15901605. https://doi.org/10.1016/j.compbiomed.2013.08.001

Chen, Y., Liu, B., O’Connor, J. M., Didier, C. S., \& Glick, S. J. (2009). Characterization of scatter in cone-beam CT breast imaging: Comparison of experimental measurements and Monte Carlo simulation. Medical Physics, 36, 857-869. https://doi.org/10.1118/1.3077122

Chen, B., Polatkan, G., Sapiro, G., Blei, D., Dunson, D., \& Carin, L. (2013). Deep learning with hierarchical convolutional factor analysis. IEEE Transactions on Pattern Analysis and Machine Intelligence, 35, 1887-1901. https://doi.org/10.1109/TPAMI.2013.19

Chou, H.-C., Cheng, C.-H., \& Chang, J.-R. (2007). Extracting drug utilization knowledge using selforganizing map and rough set theory. Expert Systems with Applications, 33, 499-508.

Chua, K. C., Chandran, V., Acharya, U. R., \& Lim, C. M. (2010). Application of higher order statistics/ spectra in biomedical signals-A review. Medical Engineering \& Physics, 32, 679-689. https:// doi.org/10.1016/j.medengphy.2010.04.009

Coletti, G., \& Scozzafava, R. (2000). The role of coherence in eliciting and handling imprecise probabilities and its application to medical diagnosis. Information Sciences, 130, 41-65. https://doi. org/10.1016/S0020-0255(00)00085-2

Coolen, F.P.A., Troffaes, M.C.M., Augustin, T. (2011). Imprecise Probability. In Lovric M (ed), International Encyclopedia of Statistical Science. Springer, pp 645-648.

Corani, G., Magli, C., Giusti, A., Gianaroli, L., \& Gambardella, L. M. (2013). A Bayesian network model for predicting pregnancy after in vitro fertilization. Computers in Biology and Medicine, 43, 1783-1792. https://doi.org/10.1016/j.compbiomed.2013.07.035

Dahl, GE., Sainath TN, Hinton GE Improving deep neural networks for LVCSR using rectified linear units and dropout. In IEEE International Conference on Acoustics, Speech and Signal Processing, 2013. pp 8609-8613

Das, H., Naik, B., \& Behera, H. S. (2020). Medical disease analysis using Neuro-Fuzzy with Feature Extraction Model for classification. Informatics in Medicine Unlocked, 18, 100288. https://doi.org/10. 1016/j.imu.2019.100288

Dash, S., \& Patra, B. (2013). Redundant gene selection based on genetic and quick-reduct algorithms. International Journal on Data Mining and Intelligent Information Technology Application, 3, 1-9.

de Medeiros, I. B., Soares Machado, M. A., Damasceno, W. J., Caldeira, A. M., dos Santos, R. C., \& da Silva Filho, J. B. (2017). A Fuzzy Inference System to Support Medical Diagnosis in Real Time. Procedia Computer Science, 122, 167-173. 
Dempster, A. P. (1968). A Generalization of Bayesian Inference. Journal of the Royal Statistical Society: Series B (Methodological), 30, 205-232. https://doi.org/10.1111/j.2517-6161.1968.tb00722.x

Denźux, T. (2016). 40 years of Dempster-Shafer theory. Int J Approx Reasoning, 79, 1-6. https://doi.org/10. 1016/j.ijar.2016.07.010

Depeweg, S., Hernandez-Lobato J.-M., Doshi-Velez, F., Udluft, S. (2018). Decomposition of Uncertainty in Bayesian Deep Learning for Efficient and Risk-sensitive Learning. Paper presented at the Proceedings of the 35th International Conference on Machine Learning

Dervishi, A. (2017). Fuzzy risk stratification and risk assessment model for clinical monitoring in the ICU. Computers in Biology and Medicine, 87, 169-178. https://doi.org/10.1016/j.compbiomed.2017.05. 034

Dilli, R., Argou, A., Reiser, R., Yamin, A. (2018). IoT Resources Ranking: Decision Making Under Uncertainty Combining Machine Learning and Fuzzy Logic. In Fuzzy Information Processing, 2018. Springer, pp 119-131

Downes, P., Jarvis, R., Radu, E., Kawrakow, I., \& Spezi, E. (2009). Monte Carlo simulation and patient dosimetry for a kilovoltage cone-beam CT unit. Medical Physics, 36, 4156-4167. https://doi.org/10. $1118 / 1.3196182$

Flügge, S., Zimmer, S., Petersohn, U. (2019). Knowledge representation and diagnostic inference using Bayesian networks in the medical discourse arXiv preprint arXiv: 190908549

Fox, R. C. (1957). Training for uncertainty. The student physician, 3, 207-241.

Fox, R. C. (1980). The Evolution of Medical Uncertainty. The Milbank Memorial Fund Quarterly Health and Society, 58, 1-49. https://doi.org/10.2307/3349705

Friston, K., Mattout, J., Trujillo-Barreto, N., Ashburner, J., \& Penny, W. (2007). Variational free energy and the Laplace approximation. NeuroImage, 34, 220-234.

Gal, Y. (2016). Uncertainty in deep learning. Cambridge: University of Cambridge.

Gal, Y., Ghahramani, Z. (2015), Bayesian convolutional neural networks with Bernoulli approximate variational inference arXiv:1-12

Gal, Y., Islam, R., Ghahramani, Z. (2017). Deep bayesian active learning with image data arXiv:1-10

Galesic, M., Gigerenzer, G., \& Straubinger, N. (2009). Natural frequencies help older adults and people with low numeracy to evaluate medical screening tests. Medical Decision Making : An International Journal of the Society For Medical Decision Making, 29, 368-371.

Gallagher, C. V., Bruton, K., Leahy, K., \& O'Sullivan, D. T. J. (2018). The suitability of machine learning to minimise uncertainty in the measurement and verification of energy savings. Energy and Buildings, $158,647-655$.

Gandhimathi, T. (2018). An application of intuitionistic fuzzy soft matrix in medical diagnosis. Journal of Computational and Theoretical Nanoscience, 15, 781-784.

Gao, J., Zhang, H., Lu, P., Wang, Z. (2019). An effective LSTM recurrent network to detect arrhythmia on imbalanced ECG dataset. Journal of Healthcare Engineering 2019

Gasparini, A., Abrams, K. R., Barrett, J. K., Major, R. W., Sweeting, M. J., Brunskill, N. J., \& Crowther, M. J. (2020). Mixed-effects models for health care longitudinal data with an informative visiting process: A Monte Carlo simulation study. Statistica Neerlandica, 74, 5-23. https://doi.org/10.1111/stan.12188

Ghasemi, J., Ghaderi, R., Karami Mollaei, M. R., \& Hojjatoleslami, S. A. (2013). A novel fuzzy DempsterShafer inference system for brain MRI segmentation. Information Sciences, 223, 205-220.

Ghassemi, N. et al. (2020). Material recognition for automated progress monitoring using deep learning methods arXiv preprint, arXiv:200616344

Ghassemi, N., Shoeibi, A., Rouhani, M., Hosseini-Nejad, H. (2019). Epileptic seizures detection in EEG signals using TQWT and ensemble learning. In 2019 9th International Conference on Computer and Knowledge Engineering (ICCKE), 24-25 Oct. 2019. pp 403-408. doi:https://doi.org/10.1109/ICCKE 48569.2019.8964826

Ghazavi, S. N., \& Liao, T. W. (2008). Medical data mining by fuzzy modeling with selected features. Artificial Intelligence in Medicine, 43, 195-206.

Ghobadi, P., Farhood, B., Ghorbani, M., \& Mohseni, M. (2020). Design and characterization of flattening filter for high dose rate 192Ir and 60Co Leipzig applicators used in skin cancer brachytherapy: A Monte Carlo study. Computers in Biology and Medicine, 123, 103878. https://doi.org/10.1016/j. compbiomed.2020.103878

Ghoshal. B., Tucker, A. (2020). Estimating uncertainty and interpretability in deep learning for coronavirus (COVID-19) detection arXiv preprint arXiv:200310769

Ghoshal. B., Tucker, A., Sanghera, B., Lup Wong. W. (2019). Estimating uncertainty in deep learning for reporting confidence to clinicians in medical image segmentation and diseases detection. Computational Intelligence $\mathrm{n} / \mathrm{a}$ doi:https://doi.org/https://doi.org/10.1111/coin.12411 
Giustinelli, P. (2020), Precise or Imprecise Probabilities? Evidence from survey response related to lateonset dementia* Pamela Giustinelli Charles F. Manski Francesca Molinari

Grzymala-Busse, J. (1988). Knowledge acquisition under uncertainty - a rough set approach. Journal of Intelligent Robotic Systems, 1, 3-16.

Hamilton, J.G., Genoff, M.C., Han, P.K.J. (2020). Health-Related Uncertainty. In: The Wiley Encyclopedia of Health Psychology. pp 305-313. doi: https://doi.org/10.1002/9781119057840.ch80

Hecquet, D., Ruskin, H. J., \& Crane, M. (2007). Optimisation and parallelisation strategies for Monte Carlo simulation of HIV infection. Computers in Biology and Medicine, 37, 691-699. https://doi.org/10. 1016/j.compbiomed.2006.06.010

Hekmat R, Goharimanesh M, Dadpour B (2020) SAT-013 Comparison Of Artificial Neural Network Model and Adaptive Neuro-Fuzzy Inference System(Anfis) Model for Predicting Hemodialysis Initiation in Methanol Poisoned Patients title here Kidney International Reports 5:S6-S7 doi:https://doi.org/10. 1016/j.ekir.2020.02.017

Henriquez, R. R., \& Korpi-Steiner, N. (2016). Bayesian inference Dilemma in medical decision-making: A need for user-friendly probabilistic reasoning tools. Clinical Chemistry, 62, 1285-1286. https://doi. org/10.1373/clinchem.2016.260935

Hérault, J., Iborra, N., Serrano, B., \& Chauvel, P. (2005). Monte Carlo simulation of a protontherapy platform devoted to ocular melanoma. Medical Physics, 32, 910-919. https://doi.org/10.1118/1.1871392

Howle, L. E., Weber, P. W., \& Nichols, J. M. (2017). Bayesian approach to decompression sickness model parameter estimation. Computers in Biology and Medicine, 82, 3-11. https://doi.org/10.1016/j.compb iomed.2017.01.006

Huang, Y., Wu, H., \& Acosta, E. P. (2010). Hierarchical Bayesian inference for HIV dynamic differential equation models incorporating multiple treatment factors. Biometrical Journal, 52, 470-486. https:// doi.org/10.1002/bimj.200900173

Huang, Y., Dagne, G., \& Wu, L. (2011). Bayesian inference on joint models of HIV dynamics for time-toevent and longitudinal data with skewness and covariate measurement errors. Statistics in Medicine, 30, 2930-2946. https://doi.org/10.1002/sim.4321

Huberts, W., Donders, W. P., Delhaas, T., \& van de Vosse, F. N. (2014). Applicability of the polynomial chaos expansion method for personalization of a cardiovascular pulse wave propagation model International Journal for Numerical Methods in Biomedical. Engineering, 30, 1679-1704. https://doi.org/ $10.1002 / \mathrm{cnm} .2695$

Jafar MN, Imran R, Riffat SHA, Shuaib R (2020) Medical diagnosis using neutrosophic soft matrices and their compliments. International Journal of Advanced Research in Computer Science 11

Jain, K., Kulkarni, S. (2020). Multi-reduct rough set classifier for computer-aided diagnosis in medical data. In Verma OP, Roy S, Pandey SC, Mittal M (eds) Advancement of Machine Intelligence in Interactive Medical Image Analysis. Springer, pp 167-183. doi:https://doi.org/10.1007/978-981-15-1100-4_7

Jeeva, J. B., \& Singh, M. (2015). Reconstruction of optical scanned images of inhomogeneities in biological tissues by Monte Carlo simulation. Computers in Biology and Medicine, 60, 92-99. https://doi.org/ 10.1016/j.compbiomed.2015.02.014

Jensen, R., \& Shen, Q. (2004). Fuzzy-rough attribute reduction with application to web categorization. Fuzzy sets and systems, 141, 469-485.

Jeong, J.-H., Yu, B.-W., Lee, D.-H., \& Lee, S.-W. (2019). Classification of Drowsiness Levels Based on a Deep Spatio-Temporal Convolutional Bidirectional LSTM Network Using Electroencephalography. Signals Brain Sci, 9, 348. https://doi.org/10.3390/brainsci9120348

Jia, X., Gu, X., Graves, Y. J., Folkerts, M., \& Jiang, S. B. (2011). GPU-based fast Monte Carlo simulation for radiotherapy dose calculation. Physics in Medicine and Biology, 56, 7017-7031.

Jiang, Q.-y, Yang, X.-j, \& Sun, X.-s. (2017). An aided diagnosis model of sub-health based on rough set and fuzzy mathematics: A case of TCM. Journal of Intelligent \& Fuzzy Systems, 32, 4135-4143. https:// doi.org/10.3233/JIFS-15958

Johnson, W. O., \& Gastwirth, J. L. (1991). Bayesian Inference for Medical Screening Tests: Approximations Useful for the Analysis of Acquired Immune Deficiency Syndrome. Journal of the Royal Statistical Society: Series B (Methodological), 53, 427-439. https://doi.org/10.1111/j.2517-6161.1991.tb018 35.x

Johnston, I. G., et al. (2015). Stochastic modelling Bayesian inference, and new in vivo measurements elucidate the debated mtDNA bottleneck mechanism. Elife 4, e07464.

Kai-Quan, S. S-rough sets and its applications in diagnosis-recognition for disease. In: Proceedings. International Conference on Machine Learning and Cybernetics, 2002. pp 50-54

Kang, B., Chhipi-Shrestha, G., Deng, Y., Mori, J., Hewage, K., \& Sadiq, R. (2018). Development of a predictive model for Clostridium difficile infection incidence in hospitals using Gaussian mixture 
model and Dempster-Shafer theory. Stochastic Environmental Research and Risk Assessment, 32, 1743-1758.

Karaboga, D., \& Kaya, E. (2019). Adaptive network based fuzzy inference system (ANFIS) training approaches: a comprehensive survey. Artificial Intelligence Review, 52, 2263-2293.

Karimifard, S., \& Ahmadian, A. (2011). A robust method for diagnosis of morphological arrhythmias based on Hermitian model of higher-order statistics. BioMedical Engineering OnLine, 10, 22. https://doi. org/10.1186/1475-925X-10-22

Kendall, A., Cipolla, R. (2016). Modelling uncertainty in deep learning for camera relocalization. In: IEEE International Conference on Robotics and Automation (ICRA). pp 4762-4769

Kendall, A., Gal, Y. (2017). What uncertainties do we need in Bayesian deep learning for computer vision? Paper presented at the NIPS

Kendall, A., Badrinarayanan, V., Cipolla, R. (2015). Bayesian segnet: Model uncertainty in deep convolutional encoder-decoder architectures for scene understanding arXiv 1-11

Khodabakhshi, M. B., \& Moradi, M. H. (2017). The attractor recurrent neural network based on fuzzy functions: An effective model for the classification of lung abnormalities. Computers in Biology and Medicine, 84, 124-136. https://doi.org/10.1016/j.compbiomed.2017.03.019

Khodatars, M. et al. (2020). Deep Learning for Neuroimaging-based Diagnosis and Rehabilitation of Autism Spectrum Disorder: A Review arXiv preprint arXiv:200701285

Koistinen, P., (2010), Monte Carlo Methods, with an emphasis on Bayesian computation Summer 2010

Korattikara, A., Rathod, V., Murphy, K., Welling, M. (2015). Bayesian dark knowledge. Paper presented at the Proceedings of the 28th International Conference on Neural Information Processing Systems, Montreal, Canada,

Kosheleva, O., Kreinovich, V. (2019), Beyond p-Boxes and Interval-Valued Moments: Natural Next Approximations to General Imprecise Probabilities

Kosko, B. (1994). Fuzzy systems as universal approximators. IEEE Transactions on Computers, 43, 1329_ 1333. https://doi.org/10.1109/12.324566

Kour, H., Manhas, J., \& Sharma, V. (2020). Usage and implementation of neuro-fuzzy systems for classification and prediction in the diagnosis of different types of medical disorders: a decade review. Artificial Intelligence Review. https://doi.org/10.1007/s10462-020-09804-X

Kourou, K., Rigas, G., Papaloukas, C., Mitsis, M., \& Fotiadis, D. I. (2020). Cancer classification from time series microarray data through regulatory Dynamic Bayesian Networks. Computers in Biology and Medicine, 116, 103577. https://doi.org/10.1016/j.compbiomed.2019.103577

Kumar, M., Stoll, R., \& Stoll, N. (2003). Regularized Adaptation of Fuzzy Inference Systems. Modelling the Opinion of a Medical Expert about Physical Fitness: An Application Fuzzy Optimization and Decision Making, 2, 317-336.

Kwiatkowska, M., Riben, P., \& Kielan, K. (2009). Interpretation of Imprecision in Medical Data. Advances in Data Management (pp. 351-369). Springer.

Laha, M., Hazra, P., Konar, A., Rakshit, P. (2019). EEG-induced Probabilistic Prediction of the ColorPathways in the Brain using Dempster-Shafer Theory. In: 2019 International Conference on Wireless Communications Signal Processing and Networking (WiSPNET), 21-23, 2019. pp 339-343. doi:https://doi.org/10.1109/WiSPNET45539.2019.9032841

Lee, Y. (2020). Preliminary evaluation of dual-head Compton camera with Si/CZT material for breast cancer detection: Monte Carlo simulation study. Optik, 202, 163519. https://doi.org/10.1016/j.ijleo.2019. 163519

Lee, C., Kim, K. P., Long, D. J., \& Bolch, W. E. (2012). Organ doses for reference pediatric and adolescent patients undergoing computed tomography estimated by Monte Carlo simulation. Medical Physics, 39, 2129-2146. https://doi.org/10.1118/1.3693052

Levis, B., et al. (2018). Probability of major depression diagnostic classification using semi-structured versus fully structured diagnostic interviews. The British Journal of Psychiatry, 212, 377-385. https:// doi.org/10.1192/bjp.2018.54

Li, Z., Wen, G., \& Xie, N. (2015). An approach to fuzzy soft sets in decision making based on grey relational analysis and Dempster-Shafer theory of evidence: An application in medical diagnosis. Artificial Intelligence in Medicine, 64, 161-171.

$\mathrm{Li}, \mathrm{T}$., et al. (2020). Analysis of medical rescue strategies based on a rough set and genetic algorithm: A disaster classification perspective. International Journal of Disaster Risk Reduction, 42, 101325. https:// doi.org/10.1016/j.ijdrr.2019.101325

Liesenfeld, R., \& Richard, J.-F. (2001). Monte Carlo methods and Bayesian computation: importance sampling. International Encyclopedia of the Social and Behavioral Sciences, 2, 10000-10004.

Lim, T. K. (2020). The facts, fallacies and uncertainties about coronavirus disease 2019 (COVID-19). Ann Acad Med Singap, 49, 343-345. 
Lima, S.A., Islam, M.R. (2019). A modified method for brain MRI segmentation using Dempster-Shafer theory. In 2019 22nd International Conference on Computer and Information Technology (ICCIT), 18-20, 2019. pp 1-6. doi:https://doi.org/10.1109/ICCIT48885.2019.9038265

Lin, H.-C., Li, H.-Y., Wu, Y.-T., Tsai, Y.-L., Chuang, C.-Y., Lin, C.-H., \& Chen, W.-Y. (2020). Bayesian inference of nonylphenol exposure for assessing human dietary risk. Science of The Total Environment, 713, 136710. https://doi.org/10.1016/j.scitotenv.2020.136710

Lipková, J., et al. (2019). Personalized Radiotherapy Design for Glioblastoma: Integrating Mathematical Tumor Models. Multimodal Scans, and Bayesian Inference IEEE Transactions on Medical Imaging, 38, 1875-1884. https://doi.org/10.1109/TMI.2019.2902044

Liu, K., et al. (2019). Big medical data decision-making intelligent system exploiting fuzzy inference logic for Prostate Cancer in developing Countries. IEEE Access, 7, 2348-2363. https://doi.org/10.1109/ ACCESS.2018.2886198

Louizos, C., Ullrich, K., Welling, M. (2017). Bayesian compression for deep learning. In Advances in Neural Information Processing Systems, pp 3288-3298

Ma, W. J., Beck, J. M., Latham, P. E., \& Pouget, A. (2006). Bayesian inference with probabilistic population codes. Nature Neuroscience, 9, 1432-1438.

Magnusson, B. P., Schmidli, H., Rouyrre, N., \& Scharfstein, D. O. (2019). Bayesian inference for a principal stratum estimand to assess the treatment effect in a subgroup characterized by postrandomization event occurrence. Statistics in Medicine, 38, 4761-4771. https://doi.org/10.1002/sim.8333

Mahmoud, A.M. (2016), Suitability of Various Intelligent Tree Based Classifiers for Diagnosing Noisy Medical Data. Egyptian Computer Science Journal 40

Majeed Alneamy, J. S., \& A. Hameed Alnaish Z, Mohd Hashim SZ, Hamed Alnaish RA, . (2019). Utilizing hybrid functional fuzzy wavelet neural networks with a teaching learning-based optimization algorithm for medical disease diagnosis. Computers in Biology and Medicine, 112, 103348. https://doi. org/10.1016/j.compbiomed.2019.103348

Mazur, D. J. (2012). A history of evidence in medical decisions: from the diagnostic sign to Bayesian inference. Medical Decision Making, 32, 227-231.

McKenna, M. T., Weis, J. A., Brock, A., Quaranta, V., \& Yankeelov, T. E. (2018). Precision Medicine with Imprecise Therapy: Computational Modeling for Chemotherapy in Breast Cancer Translational. Oncology, 11, 732-742. https://doi.org/10.1016/j.tranon.2018.03.009

Meilia, P. D. I., Freeman, M. D., \& Herkutanto, Z. M. P. (2020). A review of causal inference in forensic medicine Forensic Science. Medicine and Pathology. https://doi.org/10.1007/s12024-020-00220-9

Melie-Garcia, L., Draganski, B., Ashburner, J., Kherif, F. (2018). multiple linear regression: Bayesian inference for distributed and big data in the medical informatics platform of the human brain project. bioRxiv:242883 doi:https://doi.org/10.1101/242883

Mendoza-Blanco, J. R., Tu, X. M., \& Iyengar, S. (1996). Bayesian inference on prevalence using a missingdata approach with simulation-based techniques: applications to HIV screening. Statistics in Medicine, 15, 2161-2176.

Minka, T.P. (2001). Expectation propagation for approximate Bayesian inference. Paper presented at the Proceedings of the Seventeenth conference on Uncertainty in artificial intelligence, Seattle, Washington,

Mohammadpoor, M., Shoeibi, A., \& Shojaee, H. (2016). A hierarchical classification method for breast tumor detection Iranian Journal of. Medical Physics, 13, 261-268.

Mooney, C. Z. (1997). Monte carlo simulation (Vol. 116). Cambridge: Sage publications.

Mori, Y., Seki, H., Inuiguchi, M. (2019). Knowledge Acquisition with Deep Fuzzy Inference Model and Its Application to a Medical Diagnosis. In: IEEE 10th International Conference on Awareness Science and Technology (iCAST), 23-25 Oct. 2019. pp 1-6

Nabwey, H. A. (2020). A Methodology Based on Rough Set Theory and Hypergraph for the Prediction of Wart Treatment. International Journal of Engineering Research and Technology, 13, 552-559.

Nazri, M.Z.A., Kurniawan, R., Abdullah, S.N.H.S., Othman, Z.A., Abdullah, S. (2020). Bayesian network and dempster-shafer theory for early diagnosis of eye diseases COMPUSOFT: An International Journal of Advanced Computer Technology 9

Nguyen, T., Khosravi, A., Creighton, D., \& Nahavandi, S. (2015). Medical data classification using interval type-2 fuzzy logic system and wavelets. Applied Soft Computing, 30, 812-822.

Nguyen, T. T., Phan, T. C., Nguyen, Q. V. H., Aberer, K., \& Stantic, B. (2019). Maximal fusion of facts on the web with credibility guarantee. Information Fusion, 48, 55-66.

Ningler, M., Stockmanns, G., Schneider, G., Kochs, H.-D., \& Kochs, E. (2009). Adapted variable precision rough set approach for EEG analysis. Artificial Intelligence in Medicine, 47, 239-261.

Ocampo, E., Maceiras, M., Herrera, S., Maurente, C., Rodríguez, D., \& Sicilia, M. A. (2011). Comparing Bayesian inference and case-based reasoning as support techniques in the diagnosis of Acute 
Bacterial Meningitis. Expert Systems with Applications, 38, 10343-10354. https://doi.org/10.1016/j. eswa.2011.02.055

Oh, S. L., Ng, E. Y. K., Tan, R. S., \& Acharya, U. R. (2018). Automated diagnosis of arrhythmia using combination of CNN and LSTM techniques with variable length heart beats. Computers in Biology and Medicine, 102, 278-287.

Oh, S. L., Ng, E. Y. K., Tan, R. S., \& Acharya, U. R. (2019). Automated beat-wise arrhythmia diagnosis using modified U-net on extended electrocardiographic recordings with heterogeneous arrhythmia types. Computers in Biology and Medicine, 105, 92-101.

Ormerod, J. T., \& Wand, M. P. (2010). Explaining Variational Approximations The American Statistician, $64,140-153$.

Ornelas-Vences, C., Sanchez-Fernandez, L. P., Sanchez-Perez, L. A., Garza-Rodriguez, A., \& VillegasBastida, A. (2017). Fuzzy inference model evaluating turn for Parkinson's disease patients. Computers in Biology and Medicine, 89, 379-388. https://doi.org/10.1016/j.compbiomed.2017.08.026

Özkan, A. O., Kara, S., Salli, A., Sakarya, M. E., \& Güneş, S. (2010). Medical diagnosis of rheumatoid arthritis disease from right and left hand Ulnar artery Doppler signals using adaptive network based fuzzy inference system (ANFIS) and MUSIC method. Advances in Engineering Software, 41, 12951301. https://doi.org/10.1016/j.advengsoft.2010.10.001

P, K.A., \& Acharjya, D. P. (2020). A Hybrid Scheme for Heart Disease Diagnosis Using Rough Set and Cuckoo Search Technique. Journal of Medical Systems, 44, 27. https://doi.org/10.1007/ s10916-019-1497-9

Pandya, B.H. (2015). Image processing for movement detection and face recognition using fuzzy neural network. Kadi Sarva Vishwavidyalaya

Papadimitroulas, P., Loudos, G., Nikiforidis, G. C., \& Kagadis, G. C. (2012). A dose point kernel database using GATE Monte Carlo simulation toolkit for nuclear medicine applications: Comparison with other Monte Carlo codes. Medical Physics, 39, 5238-5247. https://doi.org/10.1118/1.4737096

Papageorgiou, E. I. (2011). A Fuzzy Inference Map approach to cope with uncertainty in modeling medical knowledge and making decisions. Intelligent Decision Technologies, 5, 219-235. https://doi.org/10. 3233/IDT-2011-0108

Parikh, C. R., Pont, M. J., \& Barrie Jones, N. (2001). Application of Dempster-Shafer theory in condition monitoring applications: a case study. Pattern Recognition Letters, 22, 777-785.

Pham, T.-H., et al. (2020). Autism Spectrum Disorder Diagnostic System Using HOS Bispectrum with EEG Signals. International Journal of Environmental Research and Public Health, 17, 971.

Pho, K.-H., Ly, S., \& Ly, S. (2019). Lukusa TM (2019) Comparison among Akaike Information Criterion. Bayesian Information Criterion and Vuong's test in Model Selection: A Case Study of Violated Speed Regulation in Taiwan, 3, 293-303.

Polat, K., \& Güneş, S. (2006). A hybrid medical decision making system based on principles component analysis, k-NN based weighted pre-processing and adaptive neuro-fuzzy inference system. Digital Signal Processing, 16, 913-921.

Porebski, S., Porwik, P., Straszecka, E., \& Orczyk, T. (2018). Liver fibrosis diagnosis support using the Dempster-Shafer theory extended for fuzzy focal elements. Engineering Applications of Artificial Intelligence, 76, 67-79.

Prameswari, E. A., Triayudi, A., \& Sholihati, I. D. (2019). Web-based E-diagnostic for Digestive System Disorders in Mumans using the Demster Shafer Method. International Journal of Computer Applications, 975, 8887.

Precharattana, M., Nokkeaw, A., Triampo, W., Triampo, D., \& Lenbury, Y. (2011). Stochastic cellular automata model and Monte Carlo simulations of CD4+ T cell dynamics with a proposed alternative leukapheresis treatment for HIV/AIDS. Computers in Biology and Medicine, 41, 546-558. https://doi. org/10.1016/j.compbiomed.2011.05.005

Priyadarshini, L., Shrinivasan, L., (2020). Design of an ANFIS based Decision Support System for Diabetes Diagnosis. In 2020 International Conference on Communication and Signal Processing (ICCSP), 2020. IEEE, pp 1486-1489

Propp, J. G., \& Wilson, D. B. (1996). Exact sampling with coupled Markov chains and applications to statistical mechanics. Random Structures \& Algorithms9, 223-252. https://doi.org/10.1002/(sici) 1098-2418(199608/09)9:1/2\%3c223::Aid-rsa14\%3e3.0.Co;2-o

Qian, Y., Bi, M., Tan, T., \& Yu, K. (2016). Very Deep Convolutional Neural Networks for Noise Robust Speech Recognition IEEE/ACM Transactions on Audio. Speech, and Language Processing, 24, 2263-2276. https://doi.org/10.1109/TASLP.2016.2602884 
Ranjit, K., Kamaldeep, K., Aditya, K., \& Divya, A. (2020). An Improved and Adaptive Approach in ANFIS to Predict Knee Diseases. International Journal of Healthcare Information Systems and Informatics (IJHISI), 15, 22-37. https://doi.org/10.4018/IJHISI.2020040102

Raza, M., Gondal, I., Green, D., \& Coppel, R. L. (2006). Fusion of FNA-cytology and gene-expression data using Dempster-Shafer Theory of evidence to predict breast cancer tumors. Bioinformation, 1, 170-175. https://doi.org/10.6026/97320630001170

Razi, S., Karami Mollaei, M. R., \& Ghasemi, J. (2019). A novel method for classification of BCI multiclass motor imagery task based on Dempster-Shafer theory. Information Sciences, 484, 14-26. https://doi.org/10.1016/j.ins.2019.01.053

Reamaroon, N., Sjoding, M. W., Lin, K., Iwashyna, T. J., \& Najarian, K. (2019). Accounting for Label Uncertainty in Machine Learning for Detection of Acute Respiratory Distress Syndrome. IEEE Journal of Biomedical and Health Informatics, 23, 407-415. https://doi.org/10.1109/JBHI.2018. 2810820

Robertson, J., DeHart, D.J. (2010). An agile and accessible adaptation of Bayesian inference to medical diagnostics for rural health extension workers. In 2010 AAAI Spring Symposium Series

Rolnick, D., Veit, A., Belongie, S., Shavit, N. (2017), Deep learning is robust to massive label noise arXiv preprint arXiv: 170510694

Rundo, L., et al. (2020). Tissue-specific and interpretable sub-segmentation of whole tumour burden on CT images by unsupervised fuzzy clustering. Computers in Biology and Medicine, 120, 103751. https://doi.org/10.1016/j.compbiomed.2020.103751

Salah, O., Ramadan, A. A., Sessa, S., Ismail, A. A., Fujie, M., \& Takanishi, A. (2013). Anfis-based sensor fusion system of sit-to-stand for elderly people assistive device protocols. International Journal of Automation and Computing, 10, 405-413.

Salgado, M. V., Penko, J., Fernandez, A., Konfino, J., Coxson, P. G., Bibbins-Domingo, K., \& Mejia, R. (2020). Projected impact of a reduction in sugar-sweetened beverage consumption on diabetes and cardiovascular disease in Argentina: A modeling study. PLoS medicine, 17, e1003224.

Santra, D., Basu, S. K., Mandal, J. K., \& Goswami, S. (2020). Rough set based lattice structure for knowledge representation in medical expert systems: Low back pain management case study. Expert Systems with Applications, 145, 113084. https://doi.org/10.1016/j.eswa.2019.113084

Sarrut, D., et al. (2014). A review of the use and potential of the GATE Monte Carlo simulation code for radiation therapy and dosimetry applications. Medical physics, 41, 064301.

Schmidt, J., Marques, M. R. G., Botti, S., \& Marques, M. A. L. (2019). Recent advances and applications of machine learning in solid-state materials science. npj Computational Materials, 5, 83. https://doi.org/10.1038/s41524-019-0221-0

Schultz, S., Krüger, J., Handels, H., \& Ehrhardt, J. (2019). Bayesian inference for uncertainty quantification in point-based deformable image registration (Vol. 10949). SPIE.

Seixas, F. L., Zadrozny, B., Laks, J., Conci, A., \& Muchaluat Saade, D. C. (2014). A Bayesian network decision model for supporting the diagnosis of dementia, Alzheimer's disease and mild cognitive impairment. Computers in Biology and Medicine, 51, 140-158. https://doi.org/10.1016/j.compb iomed.2014.04.010

Sengur, A. (2008). An expert system based on principal component analysis, artificial immune system and fuzzy k-NN for diagnosis of valvular heart diseases. Computers in Biology and Medicine, 38, 329-338. https://doi.org/10.1016/j.compbiomed.2007.11.004

Shadman Roodposhti, M., Aryal, J., Lucieer, A., \& Bryan, B. A. (2019). Uncertainty Assessment of Hyperspectral Image Classification: Deep Learning vs. Random Forest Entropy, 21, 78.

Sharifrazi, D. et al. (2020), CNN-KCL: Automatic Myocarditis Diagnosis using Convolutional Neural Network Combined with K-means Clustering

Shi, J.-Y., Shang, X.-Q., Gao, K., Zhang, S.-W., \& Yiu, S.-M. (2018). An Integrated Local Classification Model of Predicting Drug-Drug Interactions via Dempster-Shafer Theory of Evidence. Scientific Reports, 8, 11829.

Shih, T.-Y., Liu, Y.-L., Chen, H.-H., \& Wu, J. (2020). Dose evaluation of a blood irradiator using Monte Carlo simulation and MAGAT gel dosimeter Nuclear Instruments and Methods in Physics Research Section A: Accelerators, Spectrometers. Detectors and Associated Equipment, 954, 161249. https://doi.org/10.1016/j.nima.2018.09.084

Shoeibi, A., et al. (2020a). Epileptic seizure detection using deep learning techniques: A Review arXiv preprint arXiv:200701276

Shoeibi, A., et al. (2020b). Automated Detection and Forecasting of COVID-19 using Deep Learning Techniques: A Review arXiv preprint arXiv:200710785 
Shoeibi, A., et al. (2021). A comprehensive comparison of handcrafted features and convolutional autoencoders for epileptic seizures detection in EEG signals. Expert Systems with Applications, 163, 113788. https://doi.org/10.1016/j.eswa.2020.113788

Shoushtarian, M., Alizadehsani, R., Khosravi, A., Acevedo, N., McKay, C. M., Nahavandi, S., \& Fallon, J. B. (2020). Objective measurement of tinnitus using functional near-infrared spectroscopy and machine learning. PLOS ONE, 15, e0241695. https://doi.org/10.1371/journal.pone.0241695

Snoek, J., et al. (2015). Scalable Bayesian Optimization Using Deep Neural Networks. Paper presented at the Proceedings of the 32nd International Conference on Machine Learning,

Sood, S. K., Kaur, S., \& Chahal, K. K. (2020). An intelligent framework for monitoring dengue fever risk using LDA-ANFIS. Journal of Ambient Intelligence and Smart Environments, 12, 5-20. https://doi. org/10.3233/AIS-200547

Soroudi, A., \& Amraee, T. (2013). Decision making under uncertainty in energy systems: State of the art. Renewable and Sustainable Energy Reviews, 28, 376-384.

Stokić, E., Brtka, V., \& Srdić, B. (2010). The synthesis of the rough set model for the better applicability of sagittal abdominal diameter in identifying high risk patients. Computers in Biology and Medicine, 40, 786-790. https://doi.org/10.1016/j.compbiomed.2010.08.001

Straszecka, E. (2006). Combining uncertainty and imprecision in models of medical diagnosis. Information Sciences, 176, 3026-3059.

Suchard, M. A., \& Redelings, B. D. (2006). BAli-Phy: simultaneous Bayesian inference of alignment and phylogeny. Bioinformatics, 22, 2047-2048. https://doi.org/10.1093/bioinformatics/btl175

Sujatha, K., et al. (2020). Chapter 5 - Screening and early identification of microcalcifications in breast using texture-based ANFIS classification. In: Dey N, Ashour AS, James Fong S, Bhatt C (eds) Wearable and Implantable Medical Devices, vol 7. Academic Press, pp 115-140.https://doi.org/10.1016/ B978-0-12-815369-7.00005-7

Sun, X.-L., Wang, H., Li, X.-K., Cao, G.-H., Kuang, Y., \& Zhang, X.-C. (2020). Monte Carlo computer simulation of a camera system for proton beam range verification in cancer treatment. Future Generation Computer Systems, 102, 978-991. https://doi.org/10.1016/j.future.2019.09.011

Tiwari, L., Raja ,R., Sharma, V., Miri, R. (2020). Fuzzy Inference System for Efficient Lung Cancer Detection. In: Gupta M, Konar D, Bhattacharyya S, Biswas S (eds) Computer Vision and Machine Intelligence in Medical Image Analysis, Singapore, 2020// 2020. Springer Singapore, pp 33-41

Toğaçar, M., Ergen, B., \& Cömert, Z. (2020). COVID-19 detection using deep learning models to exploit Social Mimic Optimization and structured chest X-ray images using fuzzy color and stacking approaches. Computers in Biology and Medicine, 121, 103805. https://doi.org/10.1016/j.compb iomed.2020.103805

Tsai, M.-Y., et al. (2020). A new open-source GPU-based microscopic Monte Carlo simulation tool for the calculations of DNA damages caused by ionizing radiation---Part I: Core algorithm and validation. Medical Physics, 47, 1958-1970. https://doi.org/10.1002/mp.14037

Tsumoto, S. (1998). Automated extraction of medical expert system rules from clinical databases based on rough set theory. Information Sciences, 112, 67-84.

Tsumoto, S. (1998). Extraction of experts' decision rules from clinical databases using rough set model. Intelligent Data Analysis, 2, 215-227. https://doi.org/10.3233/IDA-1998-2305

Turabieh, H., Mafarja, M., \& Mirjalili, S. (2019). Dynamic Adaptive Network-Based Fuzzy Inference System (D-ANFIS) for the Imputation of Missing Data for Internet of Medical Things Applications. IEEE Internet of Things Journal, 6, 9316-9325. https://doi.org/10.1109/JIOT.2019.2926321

Van Wyk, B. E. (2020). A family-level floristic inventory and analysis of medicinal plants used in Traditional African Medicine. Journal of Ethnopharmacology, 249, 112351. https://doi.org/10.1016/j.jep. 2019.112351

Vidhya, K., \& Shanmugalakshmi, R. (2020). Modified adaptive neuro-fuzzy inference system (M-ANFIS) based multi-disease analysis of healthcare Big Data. The Journal of Supercomputing. https://doi.org/ 10.1007/s11227-019-03132-w

Wang, Y., \& Dai, Y. Chen Y.-w., Meng, F. (2015). The Evidential Reasoning Approach to Medical Diagnosis using Intuitionistic Fuzzy Dempster-Shafer Theory, International Journal of Computational Intelligence Systems, 8, 75-94.

Wang, L. L. W., \& Leszczynski, K. (2007). Estimation of the focal spot size and shape for a medical linear accelerator by Monte Carlo simulation. Medical Physics, 34, 485-488. https://doi.org/10.1118/1. 2426407

Wang, H., \& Yeung, D. (2016). Towards Bayesian Deep Learning: A Framework and Some Existing Methods. IEEE Transactions on Knowledge and Data Engineering, 28, 3395-3408. https://doi.org/10. 1109/TKDE.2016.2606428 
Wang, S.-L., Li, X., Zhang, S., Gui, J., \& Huang, D.-S. (2010). Tumor classification by combining PNN classifier ensemble with neighborhood rough set based gene reduction. Computers in Biology and Medicine, 40, 179-189.

Wang, J., Hu, Y., Xiao, F., Deng, X., \& Deng, Y. (2016). A novel method to use fuzzy soft sets in decision making based on ambiguity measure and Dempster-Shafer theory of evidence: An application in medical diagnosis. Artificial Intelligence in Medicine, 69, 1-11. https://doi.org/10.1016/j.artmed. 2016.04.004

Wang, K.-J., Chen, J.-L., \& Wang, K.-M. (2019). Medical expenditure estimation by Bayesian network for lung cancer patients at different severity stages. Computers in Biology and Medicine, 106, 97-105. https://doi.org/10.1016/j.compbiomed.2019.01.015

Watabe, T., Okuhara, Y., \& Sagara, Y. (2014). A hierarchical Bayesian framework to infer the progression level to diabetes based on deficient clinical data. Computers in Biology and Medicine, 50, 107-115. https://doi.org/10.1016/j.compbiomed.2014.04.017

Xiao, F. (2018). A Hybrid Fuzzy Soft Sets Decision Making Method in Medical Diagnosis. IEEE Access, 6, 25300-25312. https://doi.org/10.1109/ACCESS.2018.2820099

Xu, Q., Zhang, C., \& Sun, B. (2020). Emotion recognition model based on the Dempster-Shafer evidence theory. Journal of Electronic Imaging, 29, 023018.

Yadollahpour, A., Nourozi, J., Mirbagheri, S. A., Simancas-Acevedo, E., \& Trejo-Macotela, F. R. (2018). Designing and Implementing an ANFIS Based Medical Decision Support System to Predict Chronic Kidney Disease Progression. Frontiers in Physiology. https://doi.org/10.3389/fphys.2018.01753

Yang, J.-G., Kim, J.-K., Kang, U.-G., \& Lee, Y.-H. (2014). Coronary heart disease optimization system on adaptive-network-based fuzzy inference system and linear discriminant analysis (ANFIS---LDA). Personal Ubiquitous Comput, 18, 1351-1362. https://doi.org/10.1007/s00779-013-0737-0

Zadeh, L. A. (1988). Fuzzy logic Computer, 21, 83-93. https://doi.org/10.1109/2.53

Zangeneh Soroush, M., Maghooli, K., Setarehdan, S. K., \& Nasrabadi, A. M. (2019). Emotion recognition through EEG phase space dynamics and Dempster-Shafer theory. Medical Hypotheses 127 34-45. https://doi.org/10.1016/j.mehy.2019.03.025

Zhai, J.-h. (2011). Fuzzy decision tree based on fuzzy-rough technique. Soft Computing, 15, 1087-1096.

Zhang, X., Liang, F., Srinivasan, R., \& Van Liew, M. (2009). Estimating uncertainty of streamflow simulation using Bayesian neural networks. Water Resources Research. https://doi.org/10.1029/2008W R007030

Zhang, S.-W., Huang, D.-S., \& Wang, S.-L. (2010). A method of tumor classification based on wavelet packet transforms and neighborhood rough set. Computers in Biology and Medicine, 40, 430-437. https://doi.org/10.1016/j.compbiomed.2010.02.007

Zhang, Y., Sohn, K., Villegas, R., Pan,. G, Lee, H. (2015). Improving object detection with deep convolutional networks via Bayesian optimization and structured prediction. Paper presented at the CVPR,

Zhang, Q., Xie, Q., \& Wang, G. (2016). A survey on rough set theory and its applications. CAAI Transactions on Intelligence Technology, 1, 323-333.

Zhou, Q., Yu, T., Zhang, X., \& Li, J. (2020). Bayesian Inference and Uncertainty Quantification for Medical Image Reconstruction with Poisson Data. SIAM Journal on Imaging Sciences, 13, 29-52. https://doi. org/10.1137/19m 1248352

Ziasabounchi, N., \& Askerzade, I. (2014). ANFIS based classification model for heart disease prediction. International Journal of Electrical \& Computer Sciences IJECS-IJENS, 14, 7-12.

Publisher's Note Springer Nature remains neutral with regard to jurisdictional claims in published maps and institutional affiliations. 


\section{Authors and Affiliations}

Roohallah Alizadehsani ${ }^{1}$ (D) - Mohamad Roshanzamir ${ }^{2}$ - Sadiq Hussain ${ }^{3}$. Abbas Khosravi ${ }^{1}$. Afsaneh Koohestani ${ }^{1}$ - Mohammad Hossein Zangooei ${ }^{4}$. Moloud Abdar ${ }^{1}$. Adham Beykikhoshk ${ }^{5}$. Afshin Shoeibi ${ }^{6,7}$. Assef Zare ${ }^{8}$. Maryam Panahiazar $^{9} \cdot$ Saeid Nahavandi ${ }^{1}$. Dipti Srinivasan ${ }^{10} \cdot$ Amir F. Atiya $^{11}$. U. Rajendra Acharya ${ }^{12,13,14}$

1 Institute for Intelligent Systems Research and Innovations (IISRI), Deakin University, Geelong, Australia

2 Department of Computer Engineering, Faculty of Engineering, Fasa University, 74617-81189 Fasa, Iran

3 System Administrator, Dibrugarh University, Dibrugarh, Assam 786004, India

4 University of Texas At Dallas, Dallas, USA

5 Applied Artificial Intelligence Institute, Deakin University, Geelong, Australia

6 Computer Engineering Department, Ferdowsi University of Mashhad, Mashhad, Iran

7 Faculty of Electrical and Computer Engineering, Biomedical Data Acquisition Lab, K. N. Toosi University of Technology, Tehran, Iran

8 Faculty of Electrical Engineering, Gonabad Branch, Islamic Azad University, Gonabad, Iran

9 Institute for Computational Health Sciences, University of California, San Francisco, USA

10 Dept. of Electrical and Computer Engineering, National University of Singapore, Singapore 117576, Singapore

11 Department of Computer Engineering, Faculty of Engineering, Cairo University, Cairo 12613, Egypt

12 Department of Electronics and Computer Engineering, Ngee Ann Polytechnic, Singapore, Singapore

13 Department of Biomedical Engineering, School of Science and Technology, Singapore University of Social Sciences, Singapore, Singapore

14 Department of Bioinformatics and Medical Engineering, Asia University, Taichung, Taiwan 